\title{
Flat Connections and Non-Local Conserved Quantities in Irrational Conformal Field Theory f
}

\author{
M.B. Halpern \\ Department of Physics, University of California \\ Theoretical Physics Group, Lawrence Berkeley Laboratory \\ Berkeley, California 94720 \\ USA \\ N.A. Obers + \\ Centre de Physique Théorique \\ Ecole Polytechnique \\ F-91128 Palaiseau \\ France
}

\begin{abstract}
Irrational conformal field theory (ICFT) includes rational conformal field theory as a small subspace, and the affine-Virasoro Ward identities describe the biconformal correlators of ICFT. We reformulate the Ward identities as an equivalent linear partial differential system with flat connections and new non-local conserved quantities. As examples of the formulation, we solve the system of flat connections for the coset correlators, the correlators of the affine-Sugawara nests and the high-level $n$-point correlators of ICFT.
\end{abstract}

${ }^{*}$ The work of MBH was supported in part by the Director, Office of Energy Research, Office of High Energy and Nuclear Physics, Division of High Energy Physics of the U.S. Department of Energy under Contract DE-AC03-76SF00098 and in part by the National Science Foundation under grant PHY90-21139. The work of NO was supported in part by a Séjour Scientifique de Longue Durée of the Ministère des Affaires Etrangères.

†e-mail: MBHALPERN@LBL.GOV, THEORY::HALPERN

${ }^{\ddagger}$ e-mail: OBERS@ORPHEE.POLYTECHNIQUE.FR 


\section{Introduction}

Affine Lie algebra, or current algebra on the circle, was discovered independently in mathematics [1] and physics [2].

It is now understood that affine Lie algebra underlies rational conformal field theory (RCFT) and irrational conformal field theory (ICFT), which includes RCFT as a small subspace,

$$
\text { ICFT } \supset \text { RCFT . }
$$

The chiral stress tensors of ICFT live in the universal enveloping algebra of the affine algebra, where they are given by the general affine-Virasoro construction $[3,4]$,

$$
T=L^{a b}{ }_{*}^{*} J_{a} J_{b}{ }_{*}^{*}
$$

where $J_{a}, a=1, \ldots, \operatorname{dim} g$ are the currents of affine $g$ and $L^{a b}$ is a solution of the Virasoro master equation [3,4].

The solution space of the master equation includes the affine-Sugawara constructions [2,5-7] the coset constructions [2,5,8], the affine-Sugawara nests [9-11] and a vast number of new conformal field theories with generically irrational central charge. Partial classification of the solution space and other developments in the Virasoro master equation are reviewed in [12].

Recently, the present authors reported a system of equations, the affineVirasoro Ward identities [13], which describe the biconformal correlators $A(\bar{z}, z)$ of ICFT. As derived, the Ward identities have the form,

$$
\begin{aligned}
\left.\bar{\partial}_{j_{1}} \cdots \bar{\partial}_{j_{q}} \partial_{i_{1}} \cdots \partial_{i_{p}} A(\bar{z}, z)\right|_{\bar{z}=z} & =A_{g}(z) W_{j_{1} \ldots j_{q}, i_{1} \ldots i_{p}}(z) \quad, \quad A_{g}(z)=A(z, z) \\
A_{g}(z) Q_{a}^{g} & =0 \quad, \quad a=1, \ldots, \operatorname{dim} g
\end{aligned}
$$

where $W_{j_{1} \ldots j_{q}, i_{1} \ldots i_{p}}$ are the affine-Virasoro connections and $A_{g}$ is the affine-Sugawara correlator, which satisfies the KZ equation [7] and the $g$-global Ward identity in (1.3b).

The Ward identities follow from KZ-type null states, which live in the modules of the universal enveloping algebra of the affine algebra, and the affineVirasoro connections may be computed as averages in the affine-Sugawara construction on $g$. The system has been completely solved for the coset correlators 
$[13,14]$, the correlators of the affine-Sugawara nests [14] and the high-level fourpoint correlators of ICFT [14]. These developments are reviewed in Ref. 15], and relevant results will be quoted as needed in Section 4.

In this paper, we follow a suggestion of Douglas that the Ward identities might be cast in a more standard form, as a linear PDE with flat connections. We find that Douglas' intuition is correct, and an equivalent formulation of the Ward identities is the system,

$$
\begin{gathered}
\bar{\partial}_{i} A(\bar{z}, z)=A(\bar{z}, z) \bar{W}_{i}(\bar{z}, z) \quad, \quad \partial_{i} A(\bar{z}, z)=A(\bar{z}, z) W_{i}(\bar{z}, z) \\
A(\bar{z}, z) Q_{a}(\bar{z}, z)=0 \quad, \quad a=1, \ldots, \operatorname{dim} g
\end{gathered}
$$

where $\bar{W}_{i}, W_{i}$ are the flat connections of ICFT. The flat connections are nonlinear functionals of the affine-Virasoro connections, whose form we shall obtain in the development below.

Perhaps more surprising is the existence of the non-local conserved generators $Q_{a}(\bar{z}, z)$ of Lie $g$,

$$
\left[Q_{a}(\bar{z}, z), Q_{b}(\bar{z}, z)\right]=i f_{a b}^{c} Q_{c}(\bar{z}, z)
$$

which appear in (1.4b). These generators, which are also non-linear functionals of the affine-Virasoro connections, are the lift of the global generators $Q_{a}^{g}$ into the space of ICFT's: Comparing eqs.(1.3b) and (1.4b), we see that the non-local generators play the same role in the general theory - restriction of the correlators to the singlet sector of $g$ - that the global generators play in the affine-Sugawara construction. Moreover, we shall see that the non-local generators include the usual global generators of $h$,

$$
Q_{a}(\bar{z}, z)=Q_{a}^{g} \quad, \quad a \in h
$$

when $h \subset g$ is an ordinary symmetry of the construction.

The equivalence of the Ward identities (1.3) and the system of flat connections (1.4) is established in Sections 2 and 3. In Section 4, we begin to translate what is known about the affine-Virasoro connections into the language of flat connections, focusing on $S L(2, \mathbb{R})$ covariance, K-conjugation covariance, crossing symmetry and the high-level form of the flat connections on simple $g$. Using 
some of these properties, we also obtain the flat connections of the cosets and nests in Section 5.

In Section 6, we discuss our results in further detail for the coset correlators [13], obtained as usual by factorizing the biconformal correlators of $h$ and $g / h$. In this simple case, we see explicitly that flat connections and non-local conserved quantities are induced for the conformal correlators as well.

Finally, Sections 7 and 8 solve the high-level system of flat connections to obtain the high-level $n$-point biconformal and conformal correlators of ICFT. The results for the correlators exhibit induced flat connections and non-local conserved quantities for all ICFT, agree with the known form of the high-level four-point correlators [14] and continue to show good physical properties when $n \neq 4$.

\section{Flat Connections and Ward Identities}

In this section, we study a particular system of linear partial differential equations,

$$
\begin{gathered}
\bar{\partial}_{i} A(\bar{z}, z)=A(\bar{z}, z) \bar{W}_{i}(\bar{z}, z) \quad, \quad \partial_{i} A(\bar{z}, z)=A(\bar{z}, z) W_{i}(\bar{z}, z) \\
(\bar{z}, z)=\left\{\bar{z}_{1}, \ldots, \bar{z}_{n}, z_{1}, \ldots, z_{n}\right\} \\
W_{i}^{g}(z) \equiv \bar{W}_{i}(z, z)+W_{i}(z, z)
\end{gathered}
$$

under the assumption of consistency. Here $A^{\alpha}, \alpha=1, \ldots, D$ is a row vector, $\left(\bar{W}_{i}\right)_{\alpha}{ }^{\beta}$ and $\left(W_{i}\right)_{\alpha}{ }^{\beta}$ are $D \times D$ matrices, and the $\bar{z}$ 's are not necessarily complex conjugates of the $z$ 's. In what follows, we refer to $A$ and $\bar{W}_{i}, W_{i}$ as the bicorrelator and its connections respectively.

Note that the system (2.1) is not the most general PDE on $2 n$ variables. Implicit in the definition (2.1c) is the restriction that the pinched flat connections

$$
\bar{W}_{i}(z, z)=\left.\bar{W}_{i}(\bar{z}, z)\right|_{\bar{z}=z} \quad, \quad W_{i}(z, z)=\left.W_{i}(\bar{z}, z)\right|_{\bar{z}=z}
$$

are finite, where $\bar{z}=z$ means $\bar{z}_{i}=z_{i}$ for all $i=1, \ldots, n$. In this way, we exclude, for example, the $\mathrm{KZ}$ equation [7] for a $2 n$-point correlator, whose connections are singular on this line. In what follows, we refer to the quantity $W_{i}^{g}$ in $(2.1 \mathrm{c})$ as the base connection of the system. 
Proposition 2.1. The connections satisfy the flatness conditions,

$$
\begin{aligned}
& \bar{\partial}_{i} \bar{W}_{j}-\bar{\partial}_{j} \bar{W}_{i}+\left[\bar{W}_{i}, \bar{W}_{j}\right]=0 \\
& \partial_{i} W_{j}-\partial_{j} W_{i}+\left[W_{i}, W_{j}\right]=0 \\
& \left(\partial_{i}+W_{i}\right) \bar{W}_{j}=\left(\bar{\partial}_{j}+\bar{W}_{j}\right) W_{i}
\end{aligned}
$$

which say that $\bar{W}_{i}$ and $W_{i}$ are flat, and satisfy the cross-flatness condition (2.3c). Proof. These relations follow as usual, by differentiation of (2.1a).

Definition 2.2. The differential operators

$$
\overline{\mathcal{D}}_{i}(\bar{z}, z)=\bar{\partial}_{i}+\bar{W}_{i}(\bar{z}, z) \quad, \quad \mathcal{D}_{i}(\bar{z}, z)=\partial_{i}+W_{i}(\bar{z}, z)
$$

are called the covariant derivatives.

Proposition 2.3. All covariant derivatives commute,

$$
\left[\overline{\mathcal{D}}_{i}, \overline{\mathcal{D}}_{j}\right]=\left[\mathcal{D}_{i}, \mathcal{D}_{j}\right]=\left[\mathcal{D}_{i}, \overline{\mathcal{D}}_{j}\right]=0
$$

Proof. The commutativity follows immediately from Proposition 2.1.

Definition 2.4. The non-linear functionals of the flat connections,

$$
\left.W_{j_{1} \ldots j_{q}, i_{1} \ldots i_{p}} \equiv \overline{\mathcal{D}}_{j_{1}} \cdots \overline{\mathcal{D}}_{j_{q}} \mathcal{D}_{i_{1}} \cdots \mathcal{D}_{i_{p}} \mathbb{1}\right|_{\bar{z}=z}
$$

are called the connection moments, where 1 is the $D \times D$ unit matrix. As examples, we have

$$
W_{0,0}=1 \quad, \quad W_{i, 0}(z)=\bar{W}_{i}(z, z) \quad, \quad W_{0, i}(z)=W_{i}(z, z)
$$

where $\bar{W}_{i}(z, z)$ and $W_{i}(z, z)$ are the pinched flat connections in (2.2).

Proposition 2.5. The connection moments are symmetric under exchange of any pair of $i$ 's or any pair of $j$ 's.

Proof. The symmetry follows immediately from Proposition 2.3 and Definition 2.4 .

Proposition 2.6. The base connection is a flat connection,

$$
\partial_{i} W_{j}^{g}-\partial_{j} W_{i}^{g}+\left[W_{i}^{g}, W_{j}^{g}\right]=0
$$

Proof. Use (2.1c), Proposition 2.1 and the differentiation rule

$$
\partial_{i}\left[\left.f(\bar{z}, z)\right|_{\bar{z}=z}\right]=\left.\left[\left(\partial_{i}+\bar{\partial}_{i}\right) f(\bar{z}, z)\right]\right|_{\bar{z}=z} \quad .
$$


Proposition 2.7. The connection moments satisfy the consistency relations,

$$
\left(\partial_{i}+W_{i}^{g}\right) W_{j_{1} \ldots j_{q}, i_{1} \ldots i_{p}}=W_{j_{1} \ldots j_{q} i, i_{1} \ldots i_{p}}+W_{j_{1} \ldots j_{q}, i_{1} \ldots i_{p} i}
$$

Proof. Use (2.1c), (2.9), Definition 2.2, Definition 2.4 and Proposition 2.3 to verify

$$
\begin{aligned}
\text { l.h.s. }=\left(\bar{\partial}_{i}+\partial_{i}+\right. & \left.\bar{W}_{i}+W_{i}\right)\left.\overline{\mathcal{D}}_{j_{1}} \cdots \overline{\mathcal{D}}_{j_{q}} \mathcal{D}_{i_{1}} \cdots \mathcal{D}_{i_{p}} \mathbb{1}\right|_{\bar{z}=z} \\
= & \left.\left(\overline{\mathcal{D}}_{i}+\mathcal{D}_{i}\right) \overline{\mathcal{D}}_{j_{1}} \cdots \overline{\mathcal{D}}_{j_{q}} \mathcal{D}_{i_{1}} \cdots \mathcal{D}_{i_{p}} \mathbb{1}\right|_{\bar{z}=z}=\text { r.h.s. }
\end{aligned}
$$

Remark. The consistency relations (2.10) were given in eq.(8.11) of Ref. [13]. In eq.(4.1) of Ref. 114, these relations are solved to express all the connection moments in terms of the base connection and the one-sided connection moments $W_{j_{1} \ldots j_{q}, 0}$ or $W_{0, i_{1} \ldots i_{p}}$.

Definition 2.8. The base correlator $A_{g}^{\alpha}, \alpha=1, \ldots, D$ is defined as

$$
A_{g}(z) \equiv A(z, z)
$$

$\underline{\text { Proposition 2.9. }}$. The base correlator satisfies the base equation,

$$
\partial_{i} A_{g}(z)=A_{g}(z) W_{i}^{g}(z)
$$

Proof. Differentiate the base correlator with eq.(2.9), and use eq.(2.1).

Proposition 2.10. The bicorrelator satisfies the Ward identities,

$$
\begin{gathered}
\left.\bar{\partial}_{j_{1}} \cdots \bar{\partial}_{j_{q}} \partial_{i_{1}} \cdots \partial_{i_{p}} A(\bar{z}, z)\right|_{\bar{z}=z}=A_{g}(z) W_{j_{1} \ldots j_{q}, i_{1} \ldots i_{p}}(z) \\
A_{g}(z)=A(z, z) \quad, \quad \partial_{i} A_{g}(z)=A_{g}(z) W_{i}^{g}(z)
\end{gathered}
$$

Proof. Differentiate $A$ as indicated, using (2.1) and Definitions 2.4 and 2.8. Definition 2.11. The (invertible) evolution operators $\bar{F}_{\alpha}{ }^{\beta}$ and $F_{\alpha}{ }^{\beta}$ of the flat connections in (2.1a) are

$$
\begin{aligned}
& \bar{F}(\bar{z}, z) \equiv P^{*} \mathrm{e}_{z}^{\bar{z}} \mathrm{~d} \bar{z}_{i}^{\prime} \bar{W}_{i}\left(\bar{z}^{\prime}, z\right) \\
& F(\bar{z}, z) \equiv P^{*} \mathrm{e}_{\bar{z}}^{z} \mathrm{~d} z_{i}^{\prime} W_{i}\left(\bar{z}, z^{\prime}\right)
\end{aligned}
$$


where $P^{*}$ is anti-path ordering. These satisfy

$$
\begin{gathered}
\bar{\partial}_{i} \bar{F}=\bar{F} \bar{W}_{i} \quad, \quad \partial_{i} F=F W_{i} \\
\bar{F}(z, z)=F(z, z)=\mathbb{1} .
\end{gathered}
$$

Proposition 2.12. The evolution operators may be written as

$$
\begin{aligned}
& \bar{F}(\bar{z}, z)=\sum_{q=0}^{\infty} \frac{1}{q !} \sum_{j_{1} \ldots j_{q}} \prod_{\nu=1}^{q}\left(\bar{z}_{j_{\nu}}-z_{j_{\nu}}\right) W_{j_{1} \ldots j_{q}, 0}(z) \\
& F(\bar{z}, z)=\sum_{p=0}^{\infty} \frac{1}{p !} \sum_{i_{i} \ldots i_{p}} \prod_{\mu=1}^{p}\left(z_{i_{\mu}}-\bar{z}_{i_{\mu}}\right) W_{0, i_{1} \ldots i_{p}}(\bar{z})
\end{aligned}
$$

where $W_{0, i_{1} \ldots i_{p}}$ and $W_{j_{1} \ldots j_{q}, 0}$ are the one-sided connection moments.

Proof. To obtain (2.17b), follow the steps,

$$
\begin{aligned}
F(\bar{z}, z) & =F(\bar{z}, \bar{z}+z-\bar{z}) \\
& =\left.\sum_{p=0}^{\infty} \frac{1}{p !} \sum_{i_{1} \ldots i_{p}} \prod_{\mu=1}^{p}\left(z_{i_{\mu}}-\bar{z}_{i_{\mu}}\right) \partial_{i_{1}} \cdots \partial_{i_{p}} F(\bar{z}, z)\right|_{z=\bar{z}} \\
& =\left.\sum_{p=0}^{\infty} \frac{1}{p !} \sum_{i_{1} \ldots i_{p}} \prod_{\mu=1}^{p}\left(z_{i_{\mu}}-\bar{z}_{i_{\mu}}\right) \mathcal{D}_{i_{1}} \cdots \mathcal{D}_{i_{p}} 1\right|_{z=\bar{z}} \\
& =\sum_{p=0}^{\infty} \frac{1}{p !} \sum_{i_{1} \ldots i_{p}} \prod_{\mu=1}^{p}\left(z_{i_{\mu}}-\bar{z}_{i_{\mu}}\right) W_{0, i_{1} \ldots i_{p}}(\bar{z})
\end{aligned}
$$

where (2.16) and Definition 2.4 were used in the last two lines. The result in (2.17a) follows similarly from $\bar{F}(\bar{z}, z)=F(z+\bar{z}-z, z)$.

Proposition 2.13. The bicorrelator can be expressed in terms of the base correlator and the evolution operators,

$$
A(\bar{z}, z)=A_{g}(z) \bar{F}(\bar{z}, z)=A_{g}(\bar{z}) F(\bar{z}, z)
$$

Proof. These relations follow immediately from Definition 2.8 and the properties (2.16) of $\bar{F}, F$.

Definition 2.14. The (invertible) evolution operator of the base connection,

$$
A_{g}\left(z, z_{0}\right) \equiv P^{*} \mathrm{e}^{\int_{z_{0}}^{z} \mathrm{~d} z_{i}^{\prime} W_{i}^{g}\left(z^{\prime}\right)}
$$


will be called the base evolution operator. Some well-known properties of the base evolution operator include

$$
\begin{gathered}
\partial_{i} A_{g}\left(z, z_{0}\right)=A_{g}\left(z, z_{0}\right) W_{i}^{g}(z) \quad, \quad \partial_{i}^{0} A_{g}\left(z, z_{0}\right)=-W_{i}^{g}\left(z_{0}\right) A_{g}\left(z, z_{0}\right) \\
A_{g}\left(z_{0}, z_{0}\right)=1 \quad, \quad A_{g}^{-1}\left(z, z_{0}\right)=A_{g}\left(z_{0}, z\right) \\
A_{g}\left(z_{0}, z\right) A_{g}\left(\bar{z}, z_{0}\right)=A_{g}(\bar{z}, z) \\
A_{g}^{\alpha}(z)=A_{g}\left(z_{0}\right)^{\beta} A_{g}\left(z, z_{0}\right)_{\beta}{ }^{\alpha} .
\end{gathered}
$$

Proposition 2.15. The evolution operators $\bar{F}$ and $F$ are related by the base evolution operator,

$$
\bar{F}(\bar{z}, z)=A_{g}(\bar{z}, z) F(\bar{z}, z) \quad, \quad F(\bar{z}, z)=A_{g}(z, \bar{z}) \bar{F}(\bar{z}, z)
$$

Proof. Use Proposition 2.13 and (2.21b-d).

Proposition 2.16. The evolution operators $\bar{F}$ and $F$ satisfy

$$
\begin{aligned}
& \left(\partial_{i}+W_{i}^{g}(z)\right) \bar{F}(\bar{z}, z)=\bar{F}(\bar{z}, z) W_{i}(z) \\
& \left(\bar{\partial}_{i}+W_{i}^{g}(\bar{z})\right) F(\bar{z}, z)=F(\bar{z}, z) \bar{W}_{i}(\bar{z})
\end{aligned}
$$

Proof. To obtain (2.23a), use (2.21a) and (2.16a) to differentiate the first relation in (2.22). To obtain (2.23b), differentiate the second relation in (2.22).

Proposition 2.17. The flat connections can be expressed as non-linear functionals of the connection moments,

$$
\begin{aligned}
& \bar{W}_{i}=\bar{F}^{-1} \bar{\partial}_{i} \bar{F}=F^{-1}\left(\bar{\partial}_{i}+W_{i}^{g}(\bar{z})\right) F \\
& W_{i}=F^{-1} \partial_{i} F=\bar{F}^{-1}\left(\partial_{i}+W_{i}^{g}(z)\right) \bar{F}
\end{aligned}
$$

Proof. Because $\bar{F}$ and $F$ are invertible, these forms follow immediately from Definition 2.11 and Proposition 2.16.

Remark. The relations (2.24) are the inverse of eq.(2.6), which expresses the connection moments in terms of the connections. See Appendix B for further details of the inversion (2.24).

Note also that the first forms in (2.24) give $\bar{W}_{i}$ and $W_{i}$ as manifestly flat connections. The second forms in $(2.24)$ can also be used to verify the crossflatness condition $(2.3 \mathrm{c})$. 
Theorem 2.18. The system of Ward identities (2.14) is equivalent to the original system (2.1) of flat connections.

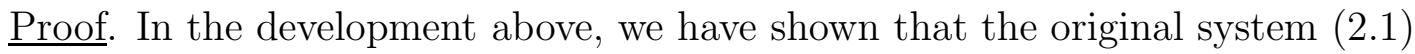
implies the Ward identities (2.14). To see that the Ward identities imply the original system, Taylor expand the correlators

$$
A(\bar{z}, z)=A(\bar{z}, \bar{z}+z-\bar{z})=A(z+\bar{z}-z, z)
$$

and use the Ward identities to obtain eqs.(2.19) in the form,

$$
\begin{aligned}
A^{\alpha}(\bar{z}, z) & =A_{g}^{\beta}(\bar{z}) \sum_{p=0}^{\infty} \frac{1}{p !} \sum_{i_{1} \ldots i_{p}} \prod_{\mu=1}^{p}\left(z_{i_{\mu}}-\bar{z}_{i_{\mu}}\right) W_{0, i_{1} \ldots i_{p}}(\bar{z})_{\beta}{ }^{\alpha} \\
& =A_{g}^{\beta}(z) \sum_{q=0}^{\infty} \frac{1}{q !} \sum_{j_{1} \ldots j_{q}} \prod_{\nu=1}^{q}\left(\bar{z}_{j_{\nu}}-z_{j_{\nu}}\right) W_{j_{1} \ldots j_{q}, 0}(z)_{\beta}{ }^{\alpha} .
\end{aligned}
$$

Then we have

$$
\begin{aligned}
& \bar{\partial}_{i} A(\bar{z}, z)=A_{g}(z) \bar{\partial}_{i} \bar{F}(\bar{z}, z)=A(\bar{z}, z) \bar{W}_{i}(\bar{z}, z) \\
& \partial_{i} A(\bar{z}, z)=A_{g}(\bar{z}) \partial_{i} F(\bar{z}, z)=A(\bar{z}, z) W_{i}(\bar{z}, z)
\end{aligned}
$$

by equation (2.16a).

Remark. The relations (2.26) were given in eq.(6.13) of Ref. 13.

\section{Non-Local Conserved Quantities}

In this section, we introduce some additional structure into the system (2.1) of flat connections. In particular, we now assume that the base connection $W_{i}^{g}$ in (2.1c) exhibits a global invariance under a Lie algebra $g$.

Definition 3.1. Let $\left(Q_{a}^{g}\right)_{\alpha}{ }^{\beta}, a=1, \ldots, \operatorname{dim} g$, called the global generators of $g$, be $D \times D$ representation matrices of Lie $g$, which satisfy

$$
\begin{gathered}
{\left[Q_{a}^{g}, W_{i}^{g}(z)\right]=0} \\
{\left[Q_{a}^{g}, Q_{b}^{g}\right]=i f_{a b}^{c} Q_{c}^{g} \quad, \quad \bar{\partial}_{i} Q_{a}^{g}=\partial_{i} Q_{a}^{g}=0}
\end{gathered}
$$

where $f_{a b}^{c}$ are the structure constants of $g$. 
Proposition 3.2. The base evolution operator in (2.20) is $g$-invariant,

$$
\left[Q_{a}^{g}, A_{g}\left(z, z_{0}\right)\right]=0
$$

Proof. The commutativity follows immediately from (3.1a) and (2.20).

Proposition 3.3. The quantities $\left(A_{g} Q_{a}^{g}\right)^{\alpha}, a=1, \ldots, \operatorname{dim} g$ are covariantly constant,

$$
\mathcal{D}_{i}^{g}\left(A_{g} Q_{a}^{g}\right) \equiv \partial_{i}\left(A_{g} Q_{a}^{g}\right)-\left(A_{g} Q_{a}^{g}\right) W_{i}^{g}=0
$$

where $A_{g}$ is the base correlator in eq. (2.12).

Proof. Differentiate as indicated, using the base equation (2.13) and eq.(3.1).

Proposition 3.4. It is consistent to choose the singlet sector of the base correlator,

$$
A_{g}(z) Q_{a}^{g}=0 \quad, \quad a=1, \ldots, \operatorname{dim} g
$$

which is called the $g$-global Ward identity. In the language of initial value problems, this means that the choice of a $g$-invariant initial condition $A_{g}\left(z_{0}\right) Q_{a}^{g}=0$ is preserved after evolution to any point $z$,

$$
A_{g}\left(z_{0}\right) Q_{a}^{g}=0 \quad \rightarrow \quad A_{g}(z) Q_{a}^{g}=0
$$

and (3.5) defines the sense in which the global generators are conserved.

Proof. The statements (3.4) and (3.5) follow immediately from Proposition 3.3.

Remark. The structure above is well known in the study of KZ equations [7], where (3.4) is called the $g$-global Ward identity. It is our task to find the lift of this structure into the system (2.1) of flat connections.

Definition 3.5. The non-linear functionals of the connection moments,

$$
Q_{a}(\bar{z}, z) \equiv F^{-1}(\bar{z}, z) Q_{a}^{g} F(\bar{z}, z) \quad, \quad a=1, \ldots, \operatorname{dim} g
$$

are called the non-local generators of $g$, where $F$ is the evolution operator in (2.15b). The explicit form of these functionals is worked out in Appendix C.

Proposition 3.6. The non-local generators are a representation of Lie $g$,

$$
\left[Q_{a}(\bar{z}, z), Q_{b}(\bar{z}, z)\right]=i f_{a b}^{c} Q_{c}(\bar{z}, z)
$$

Proof. $Q_{a}$ is a similarity transformation of $Q_{a}^{g}$. 
Proposition 3.7. The non-local generators have two equivalent forms,

$$
Q_{a}(\bar{z}, z)=F^{-1}(\bar{z}, z) Q_{a}^{g} F(\bar{z}, z)=\bar{F}^{-1}(\bar{z}, z) Q_{a}^{g} \bar{F}(\bar{z}, z)
$$

where $\bar{F}$ is the evolution operator in (2.15a).

Proof. Using Definition 3.5, Proposition 2.15 and Proposition 3.2, we have

$$
\begin{aligned}
\bar{F}^{-1}(\bar{z}, z) Q_{a}^{g} \bar{F}(\bar{z}, z) & =F^{-1}(\bar{z}, z) A_{g}^{-1}(\bar{z}, z) Q_{a}^{g} A_{g}(\bar{z}, z) F(\bar{z}, z) \\
& =F^{-1}(\bar{z}, z) Q_{a}^{g} F(\bar{z}, z) .
\end{aligned}
$$

Proposition 3.8. $Q_{a}$ is covariantly constant,

$$
\begin{aligned}
& \bar{D}_{i} Q_{a} \equiv \bar{\partial}_{i} Q_{a}+\left[\bar{W}_{i}, Q_{a}\right]=0 \\
& D_{i} Q_{a} \equiv \partial_{i} Q_{a}+\left[W_{i}, Q_{a}\right]=0 .
\end{aligned}
$$

Proof. Differentiate the two forms of $Q_{a}$ in (3.8), using eqs. (2.16a) and (3.1b).

Remark. The relations (3.10) are consistent because $W_{i}$ and $\bar{W}_{i}$ are flat and cross-flat.

Proposition 3.9. The quantities $\left(A Q_{a}\right)^{\alpha}, a=1, \ldots, \operatorname{dim} g$ are covariantly constant,

$$
\begin{aligned}
& \bar{D}_{i}\left(A Q_{a}\right)=\bar{\partial}_{i}\left(A Q_{a}\right)-\left(A Q_{a}\right) \bar{W}_{i}=0 \\
& D_{i}\left(A Q_{a}\right)=\partial_{i}\left(A Q_{a}\right)-\left(A Q_{a}\right) W_{i}=0 .
\end{aligned}
$$

Proof. These relations follow from Proposition 3.8 and the differential equations (2.1a) for the bicorrelator.

Proposition 3.10 It is consistent to choose the singlet sector of the bicorrelator,

$$
A(\bar{z}, z) Q_{a}(\bar{z}, z)=0 \quad, \quad a=1, \ldots, \operatorname{dim} g \quad .
$$

In the alternate (initial value) language of Proposition 3.4, this means that the non-local generators of $g$ are conserved quantities,

$$
A\left(\bar{z}_{0}, z_{0}\right) Q_{a}\left(\bar{z}_{0}, z_{0}\right)=0 \quad \rightarrow \quad A(\bar{z}, z) Q_{a}(\bar{z}, z)=0
$$

under the evolution defined by the system of flat connections.

Proof. The statements (3.12) and (3.13) follow immediately from Proposition 3.9 . 
Proposition 3.11. The consistent choice of singlet sectors under $Q_{a}^{g}$ and $Q_{a}(\bar{z}, z)$ are equivalent,

$$
\begin{aligned}
& A_{g}\left(z_{0}\right) Q_{a}^{g}=0 \leftrightarrow A_{g}\left(\bar{z}_{0}, z_{0}\right) Q_{a}\left(\bar{z}_{0}, z_{0}\right)=0 \\
& A_{g}(z) Q_{a}^{g}=0 \leftrightarrow A_{g}(\bar{z}, z) Q_{a}(\bar{z}, z)=0 .
\end{aligned}
$$

Proof. Using (2.19) and (3.8) we have

$$
A(\bar{z}, z) Q_{a}(\bar{z}, z)=A_{g}(z) Q_{a}^{g} \bar{F}(\bar{z}, z) \quad .
$$

Because $\bar{F}$ is invertible, the statements in (3.14) follow in both directions from eq.(3.15).

Theorem 3.12. Including the conserved quantities, the description by flat connections is equivalent to the description by Ward identities:

a) Flat connections.

$$
\begin{gathered}
\bar{\partial}_{i} A(\bar{z}, z)=A(\bar{z}, z) \bar{W}_{i}(\bar{z}, z) \quad, \quad \partial_{i} A(\bar{z}, z)=A(\bar{z}, z) W_{i}(\bar{z}, z) \\
A(\bar{z}, z) Q_{a}(\bar{z}, z)=0 \quad, \quad a=1, \ldots, \operatorname{dim} g
\end{gathered}
$$

b) Ward identities.

$$
\begin{gathered}
\left.\bar{\partial}_{j_{1}} \cdots \bar{\partial}_{j_{q}} \partial_{i_{1}} \cdots \partial_{i_{p}} A(\bar{z}, z)\right|_{\bar{z}=z}=A_{g}(z) W_{j_{1} \ldots j_{q}, i_{1} \ldots i_{p}}(z) \quad, \quad A_{g}(z)=A(z, z) \\
A_{g}(z) Q_{a}^{g}=0 \quad, a=1, \ldots, \operatorname{dim} g
\end{gathered}
$$

Proof. The equivalence of the differential systems (3.16a) and (3.17a) was established in Theorem 2.18. The equivalence of the statements of $g$-invariance in (3.16b) and (3.17b) summarizes the conclusions of this section.

\section{Irrational Conformal Field Theory}

We have shown a 1-1 correspondence between the Ward identities (3.17) and the system (3.16a) of flat connections including the non-local conserved generators of $g$ in (3.16b).

As noted in the introduction, the affine-Virasoro Ward identities [13] have exactly the form (3.17), and so irrational conformal field theory (ICFT) pro- 
vides us with a very large class of concrete realizations of the system of flat connections. In these realizations, we have the following translation dictionary. A. Bicorrelators $\rightarrow$ biconformal correlators.

Biconformal correlators are averages of Virasoro biprimary fields [16,13], which are simultaneously Virasoro primary under the commuting K-conjugate pair $[2,5,8,3]$ of affine-Virasoro constructions,

$$
\begin{aligned}
& \tilde{T}=\tilde{L}^{a b}{ }_{*}^{*} J_{a} J_{b}^{*} \quad, \quad T=L^{a b}{ }_{*}^{*} J_{a} J_{b}{ }_{*}^{*} \\
& \tilde{L}^{a b}+L^{a b}=L_{g}^{a b} \quad, \quad \tilde{T}+T=T_{g}=L_{g}^{a b *} J_{a} J_{b} * *
\end{aligned}
$$

Here, $J_{a}, a=1, \ldots, \operatorname{dim} g$ are the currents of affine $g[2,1], \tilde{L}^{a b}$ and $L^{a b}$ are a K-conjugate pair of solutions to the Virasoro master equation [3,4], and $T_{g}$ is the affine-Sugawara construction on $g$ [2,5-7].

In this paper, we discuss only the simplest set of biconformal correlators,

$$
\begin{aligned}
A^{\alpha}(\bar{z}, z) & =\left\langle R^{\alpha_{1}}\left(\mathcal{T}^{1}, \bar{z}_{1}, z_{1}\right) \ldots R^{\alpha_{n}}\left(\mathcal{T}^{n}, \bar{z}_{n}, z_{n}\right)\right\rangle \quad, \quad \alpha=\left(\alpha_{1} \ldots \alpha_{n}\right) \\
R^{\alpha}(\mathcal{T}, \bar{z}, z) & =\exp \left[(\bar{z}-z) \oint_{z} \frac{\mathrm{d} w}{2 \pi i} \tilde{T}(w)\right] R_{g}^{\alpha}(\mathcal{T}, z)=\exp \left[(z-\bar{z}) \oint_{\bar{z}} \frac{\mathrm{d} w}{2 \pi i} T(w)\right] R_{g}^{\alpha}(\mathcal{T}, \bar{z})
\end{aligned}
$$

because they satisfy the simplest affine-Virasoro Ward identities, given in (1.3). In this case, we include only the biprimary fields $R^{\alpha}(\mathcal{T}, \bar{z}, z), \alpha=1, \ldots \operatorname{dim} \mathcal{T}$ of the broken affine-primary fields, where $R_{g}^{\alpha}(\mathcal{T}, z)$ is the $L^{a b}$-broken affine primary field [13] corresponding to matrix irrep $\mathcal{T}$ of $g$.

B. Base connection $\rightarrow \mathrm{KZ}$ connection on $g$,

base equation $\rightarrow \mathrm{KZ}$ equation on $g$,

base correlator $\rightarrow$ affine-Sugawara correlator on $g$,

base evolution operator $\rightarrow$ evolution operator on $g$.

The explicit forms of these objects are well known,

$$
\begin{aligned}
& W_{i}^{g}(z)=2 L_{g}^{a b} \sum_{j \neq i}^{n} \frac{\mathcal{T}_{a}^{i} \mathcal{T}_{b}^{j}}{z_{i j}}, \quad Q_{a}^{g}=\sum_{i=1}^{n} \mathcal{T}_{a}^{i}, a=1, \ldots, \operatorname{dim} g \\
& A_{g}(z)=A(z, z)=\left\langle R_{g}\left(\mathcal{T}^{1}, z_{1}\right) \ldots R_{g}\left(\mathcal{T}^{n}, z_{n}\right)\right\rangle=A_{g}\left(z_{0}\right) A_{g}\left(z, z_{0}\right)
\end{aligned}
$$

${ }^{\dagger}$ The invariant four-point Ward identities [13] are also equivalent to a system of invariant flat connections (see Appendix A). 


$$
\partial_{i} A_{g}(z)=A_{g}(z) W_{i}^{g}(z) \quad, \quad A_{g}(z) Q_{a}^{g}=0
$$

where $\mathcal{T}_{a}^{i}, i=1, \ldots, n$ is the matrix irrep of $g$ corresponding to the $i$ th affine primary field $R_{g}$.

When $h \subset g$, we also have the flat connections and correlators of $h$,

$$
\begin{gathered}
W_{i}^{h}(z)=2 L_{h}^{a b} \sum_{j \neq i} \frac{\mathcal{T}_{a}^{i} \mathcal{T}_{b}^{j}}{z_{i j}} \quad, \quad \partial_{i} A_{h}(z)=A_{h}(z) W_{i}^{h}(z) \\
A_{h}^{\alpha}(z)=A_{h}^{\beta}\left(z_{0}\right) A_{h}\left(z, z_{0}\right)_{\beta}{ }^{\alpha} \\
{\left[Q_{a}^{g}, W_{i}^{h}(z)\right]=\left[A_{h}\left(z, z_{0}\right), Q_{a}^{g}\right]=A_{h}(z) Q_{a}^{g}=0 \quad, \quad a \in h}
\end{gathered}
$$

where $A_{h}\left(z, z_{0}\right)$ is the (invertible) evolution operator of $h$. The properties of this evolution operator mirror those of the evolution operator on $g$, including

$$
\begin{aligned}
& \partial_{i} A_{h}\left(z, z_{0}\right)=A_{h}\left(z, z_{0}\right) W_{i}^{h}(z) \quad, \quad A_{h}\left(z_{0}, z_{0}\right)=\mathbb{1} \\
& A_{h}(z, \bar{z})=A_{h}\left(z_{0}, \bar{z}\right) A_{h}\left(z, z_{0}\right)=A_{h}^{-1}\left(\bar{z}, z_{0}\right) A_{h}\left(z, z_{0}\right) \\
& A_{h}(\bar{z}, z)=A_{h}\left(z_{0}, z\right) A_{h}\left(\bar{z}, z_{0}\right)=A_{h}^{-1}\left(z, z_{0}\right) A_{h}\left(\bar{z}, z_{0}\right)
\end{aligned} .
$$

C. Connection moments $\rightarrow$ affine-Virasoro connections.

The affine-Virasoro connections $W_{j_{1} \ldots j_{q}, i_{1} \ldots i_{p}}$ may be evaluated in principle from the formula $[13,14]$,

$$
\begin{gathered}
A_{g}^{\beta}(z) W_{j_{1} \ldots j_{q}, i_{1} \ldots i_{p}}(z)_{\beta}{ }^{\alpha}= \\
{\left[\prod_{r=1}^{q} \tilde{L}^{a_{r} b_{r}} \oint_{z_{j_{r}}} \frac{\mathrm{d} \omega_{r}}{2 \pi i} \oint_{\omega_{r}} \frac{\mathrm{d} \eta_{r}}{2 \pi i} \frac{1}{\eta_{r}-\omega_{r}}\right]\left[\prod_{s=1}^{p} L^{c_{s} d_{s}} \oint_{z_{i_{s}}} \frac{\mathrm{d} \omega_{q+s}}{2 \pi i} \oint_{\omega_{q+s}} \frac{\mathrm{d} \eta_{q+s}}{2 \pi i} \frac{1}{\eta_{q+s}-\omega_{q+s}}\right]} \\
\times\left\langle J_{a_{1}}\left(\eta_{1}\right) J_{b_{1}}\left(\omega_{1}\right) \ldots J_{a_{q}}\left(\eta_{q}\right) J_{b_{q}}\left(\omega_{q}\right) J_{c_{1}}\left(\eta_{q+1}\right) J_{d_{1}}\left(\omega_{q+1}\right) \ldots\right. \\
\left.J_{c_{p}}\left(\eta_{q+p}\right) J_{d_{p}}\left(\omega_{q+p}\right) R_{g}^{\alpha_{1}}\left(\mathcal{T}^{1}, z_{1}\right) \ldots R_{g}^{\alpha_{n}}\left(\mathcal{T}^{n}, z_{n}\right)\right\rangle
\end{gathered}
$$

since the required averages are in the affine-Sugawara construction on $g$. Results have been obtained thru second order $(q+p=2)$ for all theories [13], to all orders for all coset constructions [13,14] and affine-Sugawara nests [14], and to leading order at high level for all ICFT on simple $g$ [14]. 
D. Formula for the flat connections.

Using (4.1), (4.2) and the system $\bar{\partial}_{i} A=A \bar{W}_{i}, \partial_{i} A=A W_{i}$ of flat connections, we obtain the relations

$$
\begin{aligned}
& A^{\beta}(\bar{z}, z) \bar{W}_{i}(\bar{z}, z)_{\beta}{ }^{\alpha} \\
& \quad=\oint_{\bar{z}_{i}} \frac{\mathrm{d} w}{2 \pi i} \oint_{w} \frac{\mathrm{d} \eta}{2 \pi i} \frac{\tilde{L}^{a b}}{\eta-w}\left\langle J_{a}(\eta) J_{b}(w) R^{\alpha_{1}}\left(\mathcal{T}^{1}, \bar{z}_{1}, z_{1}\right) \ldots R^{\alpha_{n}}\left(\mathcal{T}^{n}, \bar{z}_{n}, z_{n}\right)\right\rangle \\
& A^{\beta}(\bar{z}, z) W_{i}(\bar{z}, z)_{\beta}{ }^{\alpha} \\
& \quad=\oint_{z_{i}} \frac{\mathrm{d} w}{2 \pi i} \oint_{w} \frac{\mathrm{d} \eta}{2 \pi i} \frac{L^{a b}}{\eta-w}\left\langle J_{a}(\eta) J_{b}(w) R^{\alpha_{1}}\left(\mathcal{T}^{1}, \bar{z}_{1}, z_{1}\right) \ldots R^{\alpha_{n}}\left(\mathcal{T}^{n}, \bar{z}_{n}, z_{n}\right)\right\rangle
\end{aligned}
$$

which are equivalent to the formula (4.6) for the one-sided affine-Virasoro connections. The original form in (4.6) is apparently superior for computational purposes.

E. $S L(2, \mathbb{R}) \times S L(2, \mathbb{R})$ covariance.

Because affine-Virasoro constructions come in commuting K-conjugate pairs, we know that the biconformal correlators satisfy the $S L(2, \mathbb{R}) \times S L(2, \mathbb{R})$ relations,

$$
\begin{aligned}
& A \sum_{i=1}^{n} \bar{W}_{i}=A \sum_{i=1}^{n}\left(\bar{z}_{i} \bar{W}_{i}+\tilde{\Delta}_{i}\right)=A \sum_{i=1}^{n}\left(\bar{z}_{i}^{2} \bar{W}_{i}+2 \bar{z}_{i} \tilde{\Delta}_{i}\right)=0 \\
& A \sum_{i=1}^{n} W_{i}=A \sum_{i=1}^{n}\left(z_{i} W_{i}+\Delta_{i}\right)=A \sum_{i=1}^{n}\left(z_{i}^{2} W_{i}+2 z_{i} \Delta_{i}\right)=0
\end{aligned}
$$

Then, repeated differentiation of (4.8) at $\bar{z}=z$ gives the corresponding $S L(2, \mathbb{R})$ $\times S L(2, \mathbb{R})$ relations for the affine-Virasoro connections. For example, the right index or $L$-relations,

$$
\begin{gathered}
A_{g} \sum_{i=1}^{n} W_{j_{1} \ldots j_{q}, i_{1} \ldots i_{p} i}=0 \\
A_{g}\left[\sum_{i=1}^{n} z_{i} W_{j_{1} \ldots j_{q}, i_{1} \ldots i_{p} i}+\left(\sum_{i=1}^{n} \Delta_{i}+p\right) W_{j_{1} \ldots j_{q}, i_{1} \ldots i_{p}}\right]=0 \\
A_{g}\left[\sum_{i=1}^{n} z_{i}^{2} W_{j_{1} \ldots j_{q}, i_{1} \ldots i_{p} i}+2\left(\sum_{i=1}^{n} z_{i} \Delta_{i}+\sum_{\nu=1}^{p} z_{i_{\nu}}\right) W_{j_{1} \ldots j_{q}, i_{1} \ldots i_{p}}\right. \\
\left.+2 \sum_{\nu=1}^{p}\left(\Delta_{i_{\nu}}+\sum_{\mu=\nu+1}^{p} \delta_{i_{\nu}, i_{\mu}}\right) W_{j_{1} \ldots j_{q}, i_{1} \ldots \hat{i}_{\nu} . . i_{p}}\right]=0
\end{gathered}
$$


are obtained from (4.8b) using $\partial_{i} A=A W_{i}$, eq.(2.6) and the Ward identities (3.17a). In (4.9c), the omission of an index is denoted by a hat. Analogous left index or $\tilde{L}$-relations with $\Delta \rightarrow \tilde{\Delta}$ are obtained from eq.(4.8a) and $\bar{\partial}_{i} A=A \bar{W}_{i}$.

In the remainder of this section, we use the results,

$$
\begin{gathered}
\bar{W}_{i}(\bar{z}, z)=\bar{F}^{-1}(\bar{z}, z) \partial_{i} \bar{F}(\bar{z}, z) \quad, \quad W_{i}(\bar{z}, z)=F^{-1}(\bar{z}, z) \partial_{i} F(\bar{z}, z) \\
Q_{a}(\bar{z}, z)=F^{-1}(\bar{z}, z) Q_{a}^{g} F(\bar{z}, z)=\bar{F}^{-1}(\bar{z}, z) Q_{a}^{g} \bar{F}(\bar{z}, z) \\
\bar{F}(\bar{z}, z)=\sum_{q=0}^{\infty} \frac{1}{q !} \sum_{j_{1} \ldots j_{q}} \prod_{\nu=1}^{q}\left(\bar{z}_{j_{\nu}}-z_{j_{\nu}}\right) W_{j_{1} \ldots j_{q}, 0}(z) \\
F(\bar{z}, z)=\sum_{p=0}^{\infty} \frac{1}{p !} \sum_{i_{i} \ldots i_{p}} \prod_{\mu=1}^{p}\left(z_{i_{\mu}}-\bar{z}_{i_{\mu}}\right) W_{0, i_{1} \ldots i_{p}}(\bar{z})
\end{gathered}
$$

to translate some of what is known about the affine-Virasoro connections into the language of flat connections.

1. Pinched flat connections. Using (4.10) or (2.7), we obtain the pinched flat connections,

$$
\begin{gathered}
\bar{W}_{i}(z, z)=W_{i, 0}(z)=2 \tilde{L}^{a b} \sum_{j \neq i} \frac{\mathcal{T}_{a}^{i} \mathcal{T}_{b}^{j}}{z_{i j}} \\
W_{i}(z, z)=W_{0, i}(z)=2 L^{a b} \sum_{j \neq i} \frac{\mathcal{T}_{a}^{i} \mathcal{T}_{b}^{j}}{z_{i j}} \\
\bar{W}_{i}(z, z)+W_{i}(z, z)=W_{i}^{g}(z)
\end{gathered}
$$

from the known form [13] of the first-order affine-Virasoro connections.

2. $\tilde{L}$ and $L$ dependence $[13]$. The one-sided connections $W_{j_{1} \ldots j_{q}, 0}(\tilde{L}, z)$, and $W_{0, i_{1} \ldots i_{p}}(L, z)$ are functions of $\tilde{L}$ and $L$ respectively, so the evolution operators and flat connections are also functions only of $\tilde{L}$ or $L$,

$$
\begin{array}{cc}
\bar{F}(\tilde{L}, \bar{z}, z) & , \quad F(L, \bar{z}, z) \\
\bar{W}_{i}(\tilde{L}, \bar{z}, z) & , \quad W_{i}(L, \bar{z}, z)
\end{array}
$$

3. K-conjugation covariance. Under K-conjugation, the affine-Virasoro connections satisfy [13]

$$
W_{j_{1} \ldots j_{q}, 0}\left(\tilde{L}=L_{*}\right)=W_{0, j_{1} \ldots j_{q}}\left(L=L_{*}\right) \quad, \quad W_{0, i_{1} \ldots i_{p}}\left(L=L_{*}\right)=W_{i_{1} \ldots i_{p}, 0}\left(\tilde{L}=L_{*}\right)
$$


so the evolution operators and flat connections satisfy,

$$
\begin{gathered}
\bar{F}\left(\tilde{L}=L_{*}, \bar{z}, z\right)=F\left(L=L_{*}, z, \bar{z}\right) \quad, \quad F\left(L=L_{*}, \bar{z}, z\right)=\bar{F}\left(\tilde{L}=L_{*}, z, \bar{z}\right) \\
\bar{W}_{i}\left(\tilde{L}=L_{*}, \bar{z}, z\right)=W_{i}\left(L=L_{*}, z, \bar{z}\right) \quad, \quad W_{i}\left(L=L_{*}, \bar{z}, z\right)=\bar{W}_{i}\left(\tilde{L}=L_{*}, z, \bar{z}\right)
\end{gathered}
$$

From these relations, we also learn that the biconformal correlators and nonlocal conserved quantities satisfy

$$
\begin{gathered}
\left.A(\bar{z}, z)\right|_{\tilde{L} \leftrightarrow L}=A(z, \bar{z}) \\
\left.Q_{a}(\bar{z}, z)\right|_{\tilde{L} \leftrightarrow L}=Q_{a}(z, \bar{z}) \quad, \quad a=1, \ldots, \operatorname{dim} g
\end{gathered}
$$

under exchange of the K-conjugate theories.

4. Crossing symmetry. The affine-Virasoro connections satisfy the crossing symmetry 14

$$
\begin{gathered}
\left.W_{j_{1} \ldots j_{q}, i_{1} \ldots i_{p}}(z)\right|_{k \leftrightarrow l}=W_{j_{1} \ldots j_{q}, i_{1} \ldots i_{p}}(z) \\
\mathcal{T}^{k} \leftrightarrow \mathcal{T}^{l} \quad, \quad z_{k} \leftrightarrow z_{l} \quad, \quad i \leftrightarrow l \text { or } k \text { when } i=k \text { or } l
\end{gathered}
$$

where $k \leftrightarrow l$ means exchange of $\mathcal{T}$ 's, $z$ 's and indices, as given in (4.16b). It follows from (4.10) that the evolution operators, flat connections and non-local conserved quantities satisfy the crossing relations

$$
\begin{gathered}
\left.\bar{F}(\bar{z}, z)\right|_{k \leftrightarrow l}=\bar{F}(\bar{z}, z) \quad,\left.\quad F(\bar{z}, z)\right|_{k \leftrightarrow l}=F(\bar{z}, z) \\
\left.\bar{W}_{i}(\bar{z}, z)\right|_{k \leftrightarrow l}=\bar{W}_{i}(\bar{z}, z) \quad,\left.\quad W_{i}(\bar{z}, z)\right|_{k \leftrightarrow l}=W_{i}(\bar{z}, z) \\
\left.Q_{a}(\bar{z}, z)\right|_{k \leftrightarrow l}=Q_{a}(\bar{z}, z) \\
\mathcal{T}^{k} \leftrightarrow \mathcal{T}^{l}, \quad \bar{z}_{k} \leftrightarrow \bar{z}_{l} \quad, \quad z_{k} \leftrightarrow z_{l}, \quad i \leftrightarrow l \text { or } k \text { when } i=k \text { or } l
\end{gathered}
$$

where $k \leftrightarrow l$ now also includes the exchange of $\bar{z}$ 's, as given in $(4.17 \mathrm{~d})$.

5. High-level flat connections. On simple $g$, we know the high-level affineVirasoro connections [14]

$$
\begin{aligned}
& W_{j_{1} \ldots j_{q}, 0}=\left(\prod_{r=1}^{q-1} \partial_{j_{r}}\right) W_{j_{q}, 0}+\mathcal{O}\left(k^{-2}\right) \quad, \quad q \geq 1 \\
& W_{0, i_{1} \ldots i_{p}}=\left(\prod_{r=1}^{p-1} \partial_{i_{r}}\right) W_{0, i_{p}}+\mathcal{O}\left(k^{-2}\right) \quad, \quad p \geq 1
\end{aligned}
$$




$$
\begin{gathered}
W_{j_{1} \ldots j_{q}, i_{1} \ldots i_{p}}=\mathcal{O}\left(k^{-2}\right) \quad, \quad q, p \geq 1 \\
\tilde{L}^{a b}=\frac{\tilde{P}^{a b}}{2 k}+\mathcal{O}\left(k^{-2}\right) \quad, \quad L^{a b}=\frac{P^{a b}}{2 k}+\mathcal{O}\left(k^{-2}\right)
\end{gathered}
$$

where $W_{i, 0}$ and $W_{0, i}$ are given in (4.11a,b) and $\tilde{P}$ and $P$ are the high-level projectors $[17,18]$ of the $\tilde{L}$ and the $L$ theory respectively. This translates to the high-level form of the evolution operators,

$$
\begin{aligned}
& \bar{F}(\bar{z}, z)=\mathbb{1}+\frac{\tilde{P}^{a b}}{k} \sum_{i<j} \mathcal{T}_{a}^{i} \mathcal{T}_{b}^{j} \ln \left(\frac{\bar{z}_{i j}}{z_{i j}}\right)+\mathcal{O}\left(k^{-2}\right) \\
& F(\bar{z}, z)=\mathbb{1}+\frac{P^{a b}}{k} \sum_{i<j} \mathcal{T}_{a}^{i} \mathcal{T}_{b}^{j} \ln \left(\frac{z_{i j}}{\bar{z}_{i j}}\right)+\mathcal{O}\left(k^{-2}\right)
\end{aligned}
$$

and so we obtain

$$
\begin{aligned}
& \bar{W}_{i}(\bar{z}, z)=W_{i, 0}(\bar{z})+\mathcal{O}\left(k^{-2}\right)=\frac{\tilde{P}^{a b}}{k} \sum_{j \neq i} \frac{\mathcal{T}_{a}^{i} \mathcal{T}_{b}^{j}}{z_{i j}}+\mathcal{O}\left(k^{-2}\right) \\
& W_{i}(\bar{z}, z)=W_{0, i}(z)+\mathcal{O}\left(k^{-2}\right)=\frac{P^{a b}}{k} \sum_{j \neq i} \frac{\mathcal{T}_{a}^{i} \mathcal{T}_{b}^{j}}{z_{i j}}+\mathcal{O}\left(k^{-2}\right)
\end{aligned}
$$

for the high-level flat connections of ICFT.

Using (4.10b-d) and (4.19), we also obtain the high-level forms of the nonlocal conserved quantities

$$
\begin{aligned}
Q_{a}(\bar{z}, z) & =Q_{a}^{g}+\left[Q_{a}^{g}, \frac{P^{a b}}{k} \sum_{i<j} \ln \left(\frac{z_{i j}}{\bar{z}_{i j}}\right) \mathcal{T}_{a}^{i} \mathcal{T}_{b}^{j}\right]+\mathcal{O}\left(k^{-2}\right) \\
& =Q_{a}^{g}+\left[Q_{a}^{g}, \frac{\tilde{P}^{a b}}{k} \sum_{i<j} \ln \left(\frac{\bar{z}_{i j}}{z_{i j}}\right) \mathcal{T}_{a}^{i} \mathcal{T}_{b}^{j}\right]+\mathcal{O}\left(k^{-2}\right)
\end{aligned}
$$

where the equality of the two forms is easily verified with $\tilde{L}+L=L_{g}$.

In Section 7, we use these results to solve the system of flat connections for the high-level $n$-point correlators of ICFT.

\section{$5 \quad$ Flat Connections for Cosets and Nests}

The affine-Sugawara nests $[9-11,13,14]$ generalize the $g / h$ coset constructions $[2,5,8]$ to CFT's on the subgroup nests用 $g \supset h_{1} \supset \ldots \supset h_{n}$. The all-order

\footnotetext{
†The higher nests $(n \geq 2)$ are tensor product CFT's [14], but this will not be needed here.
} 
affine-Virasoro connections of the cosets and nests were computed in Ref. [14], employing an iterative scheme based on K-conjugation and the solution of the consistency relations (2.10). Using these results, we may obtain the flat connections of the cosets and nests from eq.(4.10).

Instead, we develop here a related iterative method which works directly with the flat connections. In particular, the scheme uses only the relations,

$$
\begin{gathered}
\bar{W}_{i}\left[\tilde{L}=L_{*}, \bar{z}, z\right]=W_{i}\left[L=L_{*}, z, \bar{z}\right] \\
\partial_{i} F=F W_{i} \quad, \quad F(z, z)=\mathbb{1} \\
\bar{W}_{i}(\bar{z}, z)=F^{-1}(\bar{z}, z)\left(\bar{\partial}_{i}+W_{i}^{g}(\bar{z})\right) F(\bar{z}, z)
\end{gathered}
$$

collected from above, where (5.1a) is the K-conjugation covariance of the flat connections.

$\tilde{L}=L_{g}, L=0$

For the trivial theory $L=0$, we begin with the trivial connection,

$$
W_{i}[L=0, \bar{z}, z]=0
$$

Then we may compute

$$
\begin{gathered}
F[L=0, \bar{z}, z]=\mathbb{1} \\
\bar{W}_{i}\left[\tilde{L}=L_{g}, \bar{z}, z\right]=W_{i}^{g}(\bar{z})
\end{gathered}
$$

from (5.1b) and (5.1c), where $W_{i}^{g}(z)$ is the $\mathrm{KZ}$ connection in (4.3a).

$\tilde{L}=L_{g / h}, L=L_{h}$

When $h \subset g$, we may rename the groups to obtain

$$
W_{i}\left[L=L_{h}, \bar{z}, z\right]=W_{i}^{h}(z)=2 L_{h}^{a b} \sum_{j \neq i} \frac{\mathcal{T}_{a}^{i} \mathcal{T}_{b}^{j}}{z_{i j}}
$$

from (5.3b) and (5.1a). Then, we compute from (5.1b) and (5.1c) that

$$
\begin{gathered}
F\left[L=L_{h}, \bar{z}, z\right]=A_{h}(z, \bar{z})=A_{h}^{-1}(\bar{z}, z) \\
\bar{W}_{i}\left[\tilde{L}=L_{g / h}, \bar{z}, z\right]=A_{h}(\bar{z}, z)\left(\bar{\partial}_{i}+W_{i}^{g}(\bar{z})\right) A_{h}(z, \bar{z}) \\
=A_{h}(\bar{z}, z) W_{i}^{g / h}(\bar{z}) A_{h}(z, \bar{z})
\end{gathered}
$$

where $A_{h}$ is the evolution operator (4.5) on $h$ and $W_{i}^{g / h}=W_{i}^{g}-W_{i}^{h}$. 
$\underline{\tilde{L}}=L_{g / h_{1} / h_{2}}, L=L_{h_{1} / h_{2}}$

When $g \supset h_{1} \supset h_{2}$, we obtain

$$
\begin{gathered}
W_{i}\left[L=L_{h_{1} / h_{2}}, \bar{z}, z\right]=A_{h_{2}}(z, \bar{z}) W_{i}^{h_{1} / h_{2}}(z) A_{h_{2}}(\bar{z}, z) \\
F\left[L=L_{h_{1} / h_{2}}, \bar{z}, z\right]=A_{h_{1}}(z, \bar{z}) A_{h_{2}}(\bar{z}, z) \\
\bar{W}_{i}\left[\tilde{L}=L_{g / h_{1} / h_{2}}, \bar{z}, z\right]=A_{h_{2}}(z, \bar{z}) A_{h_{1}}(\bar{z}, z) W_{i}^{g / h_{1}}(\bar{z}) A_{h_{1}}(z, \bar{z}) A_{h_{2}}(\bar{z}, z)+W_{i}^{h_{2}}(\bar{z})
\end{gathered}
$$

where $(5.6 \mathrm{a})$ follows from $(5.5 \mathrm{~b})$ and $(5.1 \mathrm{a})$, and $(5.6 \mathrm{~b}, \mathrm{c})$ are computed from (5.1b) and (5.1c) respectively.

From these examples, it is clear that we are following an iterative procedure whose general form is

$$
\begin{aligned}
\bar{W}_{i}[\tilde{L}= & \left.L_{g / h_{1} / \ldots / h_{n}}\right] \rightarrow \bar{W}_{i}\left[\tilde{L}=L_{h_{1} / \ldots / h_{n+1}}\right] \\
\rightarrow W_{i}[L & \left.=L_{h_{1} / \ldots / h_{n+1}}\right] \rightarrow F\left[L=L_{h_{1} / \ldots / h_{n+1}}\right] \\
& \rightarrow \bar{W}_{i}\left[\tilde{L}=L_{g / h_{1} / \ldots / h_{n+1}}\right] .
\end{aligned}
$$

The first step in (5.7) is a renaming of the groups, followed by the application of eqs. $(5.1 \mathrm{a}, \mathrm{b}, \mathrm{c})$ in that order.

Continuing this iteration, we find that the evolution operators of the general nest have the form

$$
\begin{aligned}
F\left[L=L_{h_{1} / \ldots / h_{2 n+1}}, \bar{z}, z\right] & =A_{h_{1}}(z, \bar{z}) A_{h_{2}}(\bar{z}, z) \cdots A_{h_{2 n+1}}(z, \bar{z}) \\
F\left[L=L_{h_{1} / \ldots / h_{2 n}}, \bar{z}, z\right] & =A_{h_{1}}(z, \bar{z}) A_{h_{2}}(\bar{z}, z) \cdots A_{h_{2 n}}(\bar{z}, z) \\
\bar{F}\left[\tilde{L}=L_{g / h_{1} / \ldots / h_{n}}, \bar{z}, z\right] & =A_{g}(\bar{z}, z) F\left[L=L_{h_{1} / \ldots / h_{n}}, \bar{z}, z\right]
\end{aligned}
$$

where $A_{h_{i}}\left(z, z_{0}\right)$ is the evolution operator on $h_{i}$, defined as shown in eq.(4.5). It follows from (5.8) and (5.1c) that the flat connections satisfy the recursion relations,

$$
\begin{aligned}
& \bar{W}_{i}\left[\tilde{L}=L_{g / h_{1} / \ldots / h_{2 n+1}}, \bar{z}, z\right] \\
& =A_{h_{2 n+1}}(\bar{z}, z)\left(\bar{W}_{i}(\bar{z}, z)\left[\tilde{L}=L_{g / h_{1} / \ldots / h_{2 n}}\right]-W_{i}^{h_{2 n+1}}(\bar{z})\right) A_{h_{2 n+1}}(z, \bar{z}) \\
& \bar{W}_{i}\left[\tilde{L}=L_{g / h_{1} / \ldots / h_{2 n}}, \bar{z}, z\right] \\
& =A_{h_{2 n}}(z, \bar{z}) \bar{W}_{i}(\bar{z}, z)\left[\tilde{L}=L_{g / h_{1} / \ldots / h_{2 n-1}}\right] A_{h_{2 n}}(\bar{z}, z)+W_{i}^{h_{2 n}}(\bar{z})
\end{aligned}
$$


which generate the flat nest connections to any desired depth, starting from eq.(5.3b).

We remark that these connections can also be obtained in an alternate iteration scheme based on the relations

$$
\begin{gathered}
\bar{W}_{i}\left[\tilde{L}=L_{*}, \bar{z}, z\right]=W_{i}\left[L=L_{*}, z, \bar{z}\right] \\
\left(\partial_{i}+W_{i}\right) \bar{W}_{j}=\left(\bar{\partial}_{j}+\bar{W}_{j}\right) W_{i} \\
\bar{W}_{i}(\tilde{L}, z, z)=W_{i, 0}(\tilde{L}, z) \quad, \quad W_{i}(L, z, z)=W_{0, i}(L, z) \quad .
\end{gathered}
$$

In this scheme, one solves the cross-flatness condition (5.10b) for $\bar{W}_{i}$ at each depth of $g / h_{1} / \ldots / h_{n}$, fixing the constants of integration by the boundary conditions (5.10c).

As a check on these results, we compute the biconformal nest correlators, using eqs.(2.19), (4.5) and the evolution operators in (5.8). We obtain

$$
\begin{aligned}
A & {\left[\tilde{L}=L_{g / h_{1} / \ldots / h_{2 n+1}}, L=L_{h_{1} / \ldots / h_{2 n+1}}\right] } \\
& =A_{g}(\bar{z}) A_{h_{1}}(z, \bar{z}) A_{h_{2}}(\bar{z}, z) \cdots A_{h_{2 n+1}}(z, \bar{z}) \\
& =A_{g}\left(z_{0}\right)\left[A_{g / h_{1}}\left(\bar{z}, z_{0}\right) A_{h_{1} / h_{2}}\left(z, z_{0}\right) \cdots A_{h_{2 n} / h_{2 n+1}}\left(\bar{z}, z_{0}\right) A_{h_{2 n+1}}\left(z, z_{0}\right)\right] \\
A & {\left[\tilde{L}=L_{g / h_{1} / \ldots / h_{2 n}}, L=L_{h_{1} / \ldots / h_{2 n}}\right] } \\
& =A_{g}(\bar{z}) A_{h_{1}}(z, \bar{z}) A_{h_{2}}(\bar{z}, z) \cdots A_{h_{2 n}}(\bar{z}, z) \\
& =A_{g}\left(z_{0}\right)\left[A_{g / h_{1}}\left(\bar{z}, z_{0}\right) A_{h_{1} / h_{2}}\left(z, z_{0}\right) \cdots A_{h_{2 n-1} / h_{2 n}}\left(z, z_{0}\right) A_{h_{2 n}}\left(\bar{z}, z_{0}\right)\right]
\end{aligned}
$$

where the definition

$$
A_{h_{i} / h_{i+1}}\left(z, z_{0}\right) \equiv A_{h_{i}}\left(z, z_{0}\right) A_{h_{i+1}}^{-1}\left(z, z_{0}\right)
$$

is used in (5.11c) and (5.11f). The results in $(5.11 \mathrm{c}, \mathrm{f})$ are precisely those obtained in Ref. [14], while the alternate forms in $(5.11 \mathrm{~b}, \mathrm{e})$ show explicitly that the biconformal correlators are independent of the reference point $z_{0}$ [14].

We turn now to the non-local conserved quantities $Q_{a}=F^{-1} Q_{a}^{g} F$ of the cosets and nests. In the form $(5.11 \mathrm{c}, \mathrm{f})$, the biconformal nest correlators satisfy the non-local conservation laws

$$
A(\bar{z}, z) Q_{a}(\bar{z}, z)=0 \quad, \quad a=1, \ldots, \operatorname{dim} g
$$


so long as the affine-Sugawara correlator $A_{g}^{\alpha}\left(z_{0}\right)$ satisfies the global Ward identity $A_{g}\left(z_{0}\right) \sum_{i=1}^{n} \mathcal{T}_{a}^{i}=0$. In further detail, if one considers only the differential system (3.16a) of flat connections, the solutions for the biconformal nest correlators are those in $(5.11 \mathrm{c}, \mathrm{f})$, with $A_{g}\left(z_{0}\right)$ replaced by an arbitrary initial condition $A\left(z_{0}\right)$. The initial condition is then fixed to $A_{g}\left(z_{0}\right)$ by requiring the non-local conservation law (5.13).

We also remark that the non-local generators $Q_{a}, a \in h$ simplify to the usual global generators of $h$ when $h \subset g$ is an ordinary symmetry of the construction. To see this, note first that

$$
\begin{gathered}
{\left[Q_{a}^{g}, W_{j}^{h_{i}}(z)\right]=\left[Q_{a}^{g}, A_{h_{i}}(\bar{z}, z)\right]=0 \quad, \quad a \in h_{k} \quad, \quad k \geq i} \\
{\left[Q_{a}^{g}, W_{j}^{h_{i}}(z)\right]=\left[Q_{a}^{g}, A_{h_{i}}(\bar{z}, z)\right]=0 \quad, \quad a \in h_{n} \quad .} \\
g \supset h_{1} \supset \ldots \supset h_{n} \quad, \quad g \equiv h_{0}
\end{gathered}
$$

where (5.14a) follows from the embedding order of the subgroups and (5.14b) is included in (5.14a). It follows from (5.8), (4.10b) and (5.14b) that

$$
\begin{gathered}
\tilde{L}=L_{g / h_{1} / \ldots / h_{n}} \quad, \quad L=L_{h_{1} / \ldots / h_{n}} \\
Q_{a}(\bar{z}, z)=Q_{a}^{g} \quad, \quad a \in h_{n}
\end{gathered}
$$

while the other generators $Q_{a}(\bar{z}, z), a \in g / h_{n}$ remain generically non-local. The coordinate-independent form of the $h$ generators in $(5.15 \mathrm{~b})$ is in accord with the known Lie $h$-invariance of the cosets and nests, and should persist for all Lie $h$-invariant conformal field theories [11].

\section{Induction to Coset CFT's}

The coset correlators [13], which describe the coset CFT's, are obtained by factorization $[13,14]$ from the biconformal correlators of $h$ and the $g / h$ coset constructions. In this section, we note that the flat connections and non-local conserved quantities of the biconformal correlators induce related flat connections and non-local conserved quantities for the coset CFT's themselves.

To warm up, we consider first the case of the affine-Sugawara construction on $g$, which is K-conjugate to the trivial theory. In this case, the results of 
Section 5 reduce to

$$
\begin{gathered}
\tilde{L}=L_{g} \quad, \quad L=0 \\
\bar{W}_{i}(\bar{z}, z)=W_{i}^{g}(\bar{z}) \quad, \quad W_{i}(\bar{z}, z)=0 \\
\bar{\partial}_{i} A(\bar{z}, z)=A(\bar{z}, z) W_{i}^{g}(\bar{z}) \quad, \quad \partial_{i} A(\bar{z}, z)=0 \\
A(\bar{z}, z)=A_{g}(\bar{z}) \\
Q_{a}(\bar{z}, z)=Q_{a}^{g}=\sum_{i=1}^{n} \mathcal{T}_{a}^{i} \quad, \quad a=1, \ldots, \operatorname{dim} g
\end{gathered}
$$

so that the biconformal correlator is the affine-Sugawara correlator on $g$ and $A(\bar{z}, z) Q_{a}(\bar{z}, z)=0$ is the usual $g$-global Ward identity. At the risk of belaboring the simplest case, this example shows clearly that the KZ equations are included in the general system (3.16) of flat connections, and that the general system is a large set of generalized KZ equations.

For $h$ and the $g / h$ coset constructions, the results of Section 5 reduce to

$$
\begin{gathered}
\tilde{L}=L_{g / h} \quad, \quad L=L_{h} \\
\bar{W}_{i}(\bar{z}, z)=A_{h}(\bar{z}, z) W_{i}^{g / h}(\bar{z}) A_{h}(z, \bar{z}) \quad, \quad W_{i}(\bar{z}, z)=W_{i}^{h}(z) \\
A^{\alpha}(\bar{z}, z)=A_{g / h}^{\beta}\left(\bar{z}, z_{0}\right) A_{h}\left(z, z_{0}\right)_{\beta}{ }^{\alpha} \\
A_{g / h}^{\alpha}\left(\bar{z}, z_{0}\right) \equiv A_{g}^{\beta}\left(z_{0}\right) A_{g / h}\left(\bar{z}, z_{0}\right)_{\beta}{ }^{\alpha} \\
Q_{a}(\bar{z}, z)=\left\{\begin{array}{c}
Q_{a}^{g}, a \in h \\
A_{h}(\bar{z}, z) Q_{a}^{g} A_{h}(z, \bar{z}) \quad, \quad a \in g / h
\end{array}\right.
\end{gathered}
$$

where $A_{g / h}^{\alpha}$ in $(6.2 \mathrm{~d})$ are the coset correlators [13], whose corresponding coset blocks $[19,13,20]$ were first discussed by Douglas.

In this case, we remark first that the coset correlators are known to satisfy the coset equations [13,

$$
\begin{aligned}
\bar{\partial}_{i} A_{g / h}^{\alpha}\left(\bar{z}, z_{0}\right) & =A_{g / h}^{\beta}\left(\bar{z}, z_{0}\right) W_{i}\left[g / h, \bar{z}, z_{0}\right]_{\beta}{ }^{\alpha} \\
W_{i}\left[g / h, \bar{z}, z_{0}\right] & =A_{h}\left(\bar{z}, z_{0}\right) W_{i}^{g / h}(\bar{z}) A_{h}^{-1}\left(\bar{z}, z_{0}\right)
\end{aligned}
$$

where the dressed coset connections $W_{i}[g / h]$ in $(6.3 \mathrm{~b})$ are flat connections. Comparing the flat connections in $(6.2 \mathrm{~b})$ and $(6.3 \mathrm{~b})$, we see that the dressed coset 
connections are induced from the barred flat connections by choosing $z$ to be the regular reference point $z_{0}$,

$$
W_{i}\left[g / h, \bar{z}, z_{0}\right]=\bar{W}_{i}\left[\tilde{L}=L_{g / h}, \bar{z}, z_{0}\right]
$$

See Section 8 for further remarks on induced flat connections for the correlators of all ICFT.

Our second remark is that the coset correlators also enjoy a set of non-local conserved generators of $g$, induced from the non-local conserved generators of the biconformal correlators. To see the induction, we need the identity

$$
A_{h}\left(z, z_{0}\right) Q_{a}(\bar{z}, z)=Q_{a}\left(\bar{z}, z_{0}\right) A_{h}\left(z, z_{0}\right)
$$

which follows from the properties $(4.5 \mathrm{~b}, \mathrm{c})$ of $A_{h}$. Then, beginning with the non-local identity for the biconformal correlator, we have

$$
\begin{aligned}
0 & =A_{g / h}^{\gamma}\left(\bar{z}, z_{0}\right) A_{h}\left(z, z_{0}\right)_{\gamma}{ }^{\beta} Q_{a}(\bar{z}, z)_{\beta}{ }^{\alpha} \\
& =A_{g / h}^{\gamma}\left(\bar{z}, z_{0}\right) Q_{a}\left(\bar{z}, z_{0}\right)_{\gamma}{ }^{\beta} A_{h}\left(z, z_{0}\right)_{\beta}{ }^{\alpha} .
\end{aligned}
$$

This tells us that the induced generators $Q_{a}\left(\bar{z}, z_{0}\right)$ are non-local conserved generators of $g$ for the coset correlators,

$$
\begin{gathered}
A_{g / h}^{\beta}\left(\bar{z}, z_{0}\right) Q_{a}\left(\bar{z}, z_{0}\right)_{\beta}{ }^{\alpha}=0 \\
{\left[Q_{a}\left(\bar{z}, z_{0}\right), Q_{b}\left(\bar{z}, z_{0}\right)\right]=i f_{a b}{ }^{c} Q_{c}\left(\bar{z}, z_{0}\right)} \\
Q_{a}\left(\bar{z}, z_{0}\right)=\left\{\begin{array}{cc}
Q_{a}^{g} & , a \in h \\
A_{h}\left(\bar{z}, z_{0}\right) Q_{a}^{g} A_{h}\left(z_{0}, \bar{z}\right) & , a \in g / h
\end{array}\right.
\end{gathered}
$$

because $A_{h}$ is invertible. The result (6.7) can also be verified directly from the form of the coset correlator in $(6.2 \mathrm{~d})$.

As anticipated, eq.(6.7) shows the well-known global $h$-invariance of the coset correlators [13], supplemented now with the new non-local conserved generators of $g / h$. 


\section{The High-Level Biconformal Correlators of ICFT}

Collecting the high-level results from Section 4, we wish to solve the high-level system of flat connections,

$$
\begin{array}{r}
\bar{\partial}_{i} A(\bar{z}, z)=A(\bar{z}, z)\left(\frac{\tilde{P}^{a b}}{k} \sum_{j \neq i} \frac{\mathcal{T}_{a}^{i} \mathcal{T}_{b}^{j}}{\bar{z}_{i j}}+\mathcal{O}\left(k^{-2}\right)\right) \\
\partial_{i} A(\bar{z}, z)=A(\bar{z}, z)\left(\frac{P^{a b}}{k} \sum_{j \neq i} \frac{\mathcal{T}_{a}^{i} \mathcal{T}_{b}^{j}}{z_{i j}}+\mathcal{O}\left(k^{-2}\right)\right) \\
A(\bar{z}, z) Q_{a}(\bar{z}, z)=\mathcal{O}\left(k^{-2}\right) \\
Q_{a}(\bar{z}, z)=Q_{a}^{g}+\left[Q_{a}^{g}, \frac{P^{a b}}{k} \sum_{i<j} \mathcal{T}_{a}^{i} \mathcal{T}_{b}^{j} \ln \left(\frac{z_{i j}}{\bar{z}_{i j}}\right)\right]+\mathcal{O}\left(k^{-2}\right) \\
=Q_{a}^{g}+\left[Q_{a}^{g}, \frac{\tilde{P}^{a b}}{k} \sum_{i<j} \mathcal{T}_{a}^{i} \mathcal{T}_{b}^{j} \ln \left(\frac{\bar{z}_{i j}}{z_{i j}}\right)\right]+\mathcal{O}\left(k^{-2}\right)
\end{array}
$$

where $\tilde{P}$ and $P$ are the high-level projectors of the $\tilde{L}$ and the $L$ theory respectively. The solutions of this system are the high-level $n$-point biconformal correlators of ICFT,

$$
\begin{gathered}
A(\bar{z}, z)=A_{g}\left(z_{0}\right)\left(\mathbb{1}+\sum_{i<j} \mathcal{T}_{a}^{i} \mathcal{T}_{b}^{j}\left[\frac{P^{a b}}{k} \ln \left(\frac{z_{i j}}{z_{i j}^{0}}\right)+\frac{\tilde{P}^{a b}}{k} \ln \left(\frac{\bar{z}_{i j}}{z_{i j}^{0}}\right)\right]+\mathcal{O}\left(k^{-2}\right)\right) \\
A\left(z_{0}, z_{0}\right)=A_{g}\left(z_{0}\right) \quad, \quad A_{g}\left(z_{0}\right) Q_{a}^{g}=0
\end{gathered}
$$

where $z_{0}$ is a regular reference point.

In further detail, the solution to the differential system $(7.1 \mathrm{a}, \mathrm{b})$ gives $(7.2 \mathrm{a})$ with the left factor as an undetermined row vector $A^{\beta}\left(z_{0}\right)$, instead of $A_{g}^{\beta}\left(z_{0}\right)$. The row vector is then fixed to be the affine-Sugawara correlator $A_{g}\left(z_{0}\right)$ by the non-local conservation law (7.1c).

Here is a partial list of the properties of the high-level biconformal correlators in (7.2). 
A. An alternate derivation. The same result (7.2) is obtained by summing the partially-factorized form of the biconformal correlators [14]

$$
\begin{aligned}
& A^{\alpha}(\bar{z}, z)= \\
& \quad \sum_{q, p=0}^{\infty} \frac{1}{q !} \sum_{j_{1} \ldots j_{q}}^{n} \frac{1}{p !} \sum_{i_{1} \ldots i_{p}}^{n} \prod_{\mu=1}^{q}\left(\bar{z}_{j_{\mu}}-z_{j_{\mu}}^{0}\right)\left[A_{g}^{\beta}\left(z_{0}\right) W_{j_{1} \ldots j_{q}, i_{1} \ldots i_{p}}\left(z_{0}\right)_{\beta}{ }^{\alpha}\right] \prod_{\nu=1}^{p}\left(z_{i_{\nu}}-z_{i_{\nu}}^{0}\right)
\end{aligned}
$$

using the high-level form of the affine-Virasoro connections in (4.18).

B. $z_{0}$-independence. The high-level biconformal correlators (7.2) are independent of the reference point $z_{0}$,

$$
\frac{\partial}{\partial z_{i}^{0}} A(\bar{z}, z)=\mathcal{O}\left(k^{-2}\right)
$$

as they should be. To see this, we may use the high-level form of the KZ equation

$$
\frac{\partial}{\partial z_{i}^{0}} A_{g}\left(z_{0}\right)=A_{g}\left(z_{0}\right)\left(\frac{(\tilde{P}+P)^{a b}}{k} \sum_{j \neq i} \frac{\mathcal{T}_{a}^{i} \mathcal{T}_{b}^{j}}{z_{i j}^{0}}+\mathcal{O}\left(k^{-2}\right)\right)
$$

or we may rearrange the result in the equivalent forms,

$$
\begin{aligned}
A(\bar{z} . z) & =A_{g}(z)\left[\mathbb{1}+\frac{\tilde{P}^{a b}}{k} \sum_{i<j} \mathcal{T}_{a}^{i} \mathcal{T}_{b}^{j} \ln \left(\frac{\bar{z}_{i j}}{z_{i j}}\right)\right]+\mathcal{O}\left(k^{-2}\right) \\
& =A_{g}(\bar{z})\left[\mathbb{1}+\frac{P^{a b}}{k} \sum_{i<j} \mathcal{T}_{a}^{i} \mathcal{T}_{b}^{j} \ln \left(\frac{z_{i j}}{\bar{z}_{i j}}\right)\right]+\mathcal{O}\left(k^{-2}\right)
\end{aligned}
$$

which also follow from eqs.(2.19) and (4.19).

C. $S L(2, \mathbb{R}) \times S L(2, \mathbb{R})$ covariance. Using the global Ward identity $(7.2 \mathrm{~b})$ and the conformal weights of the broken affine primary fields 13

$$
\left(\tilde{L}^{a b} \mathcal{T}_{a} \mathcal{T}_{b}\right)_{\alpha}{ }^{\beta}=\delta_{\alpha}^{\beta} \tilde{\Delta}_{\alpha} \quad, \quad\left(L^{a b} \mathcal{T}_{a} \mathcal{T}_{b}\right)_{\alpha}{ }^{\beta}=\delta_{\alpha}^{\beta} \Delta_{\alpha}
$$

we verify that the biconformal correlators satisfy the $S L(2, \mathbb{R}) \times S L(2, \mathbb{R})$ relations,

$$
\begin{aligned}
\sum_{i=1}^{n} \bar{\partial}_{i} A^{\alpha} & =\sum_{i=1}^{n}\left(\bar{z}_{i} \bar{\partial}_{i}+\tilde{\Delta}_{\alpha_{i}}\right) A^{\alpha}=\sum_{i=1}^{n}\left(\bar{z}_{i}^{2} \bar{\partial}_{i}+2 \bar{z}_{i} \tilde{\Delta}_{\alpha_{i}}\right) A^{\alpha}=\mathcal{O}\left(k^{-2}\right) \\
\sum_{i=1}^{n} \partial_{i} A^{\alpha} & =\sum_{i=1}^{n}\left(z_{i} \partial_{i}+\Delta_{\alpha_{i}}\right) A^{\alpha}=\sum_{i=1}^{n}\left(z_{i}^{2} \partial_{i}+2 z_{i} \Delta_{\alpha_{i}}\right) A^{\alpha}=\mathcal{O}\left(k^{-2}\right)
\end{aligned}
$$


in accord with eq.(4.8).

D. Factorized form. The biconformal correlators are easily written in the factorized form

$$
\begin{aligned}
A(\bar{z}, z)=A_{g}\left(z_{0}\right) & {\left[\mathbb{1}+\frac{\tilde{P}^{a b}}{k} \sum_{i<j} \mathcal{T}_{a}^{i} \mathcal{T}_{b}^{j} \ln \left(\frac{\bar{z}_{i j}}{z_{i j}^{0}}\right)\right] } \\
\times & {\left[\mathbb{1}+\frac{P^{a b}}{k} \sum_{i<j} \mathcal{T}_{a}^{i} \mathcal{T}_{b}^{j} \ln \left(\frac{z_{i j}}{z_{i j}^{0}}\right)\right]+\mathcal{O}\left(k^{-2}\right) }
\end{aligned}
$$

since the correction terms are $\mathcal{O}\left(k^{-2}\right)$. Using this result, we may obtain the high-level conformal correlators $\bar{A}\left(\bar{z}, z_{0}\right)$ from the factorization [13]

$$
A^{\alpha}(\bar{z}, z)=\bar{A}^{\beta}\left(\bar{z}, z_{0}\right) A\left(z, z_{0}\right)_{\beta}{ }^{\alpha}+\mathcal{O}\left(k^{-2}\right) .
$$

These correlators are given and discussed in the following section.

\section{The High-Level Conformal Correlators of ICFT}

Comparing the factorized forms (7.9) and (7.10) of the biconformal correlators, we read off the high-level $n$-point conformal correlators of ICFT,

$$
\begin{gathered}
\bar{A}\left(\bar{z}, z_{0}\right)=A_{g}\left(z_{0}\right)\left[\mathbb{1}+\frac{\tilde{P}^{a b}}{k} \sum_{i<j} \mathcal{T}_{a}^{i} \mathcal{T}_{b}^{j} \ln \left(\frac{\bar{z}_{i j}}{z_{i j}^{0}}\right)\right]+\mathcal{O}\left(k^{-2}\right) \\
\tilde{L}^{a b}=\frac{\tilde{P}^{a b}}{2 k}+\mathcal{O}\left(k^{-2}\right) .
\end{gathered}
$$

Here is a partial list of properties of these correlators.

A. High-level cosets and nests. Using the high-level forms of the evolution operators of $g$ and $h$,

$$
\begin{aligned}
& A_{g}\left(z, z_{0}\right)=\mathbb{1}+\frac{P_{g}^{a b}}{k} \sum_{i<j} \mathcal{T}_{a}^{i} \mathcal{T}_{b}^{j} \ln \left(\frac{z_{i j}}{z_{i j}^{0}}\right)+\mathcal{O}\left(k^{-2}\right) \\
& A_{h}^{-1}\left(z, z_{0}\right)=\mathbb{1}-\frac{P_{h}^{a b}}{k} \sum_{i<j} \mathcal{T}_{a}^{i} \mathcal{T}_{b}^{j} \ln \left(\frac{z_{i j}}{z_{i j}^{0}}\right)+\mathcal{O}\left(k^{-2}\right)
\end{aligned}
$$


we verify that the high-level forms of the coset correlators $(6.2 \mathrm{~d})$ are correctly included in (8.1) when $\tilde{P}=P_{g / h}=P_{g}-P_{h}$. Similarly, the high-level form of the nest correlators are obtained from (8.1) when

$$
\tilde{P}=P_{g / h_{1} / \ldots / h_{n}}=P_{g}+\sum_{j=1}^{n}(-)^{j} P_{h_{j}}
$$

and we have checked that the four-point conformal blocks of these correlators agree with the nest conformal blocks obtained in Ref.[14].

B. Induced flat connections for ICFT. It was suggested in Ref. 113 that the $n$ point correlators of ICFT might satisfy a PDE with flat connections,

$$
\bar{\partial}_{i} \bar{A}=\bar{A} W_{i}[\tilde{L}] \quad, \quad W_{i}[\tilde{L}]=\frac{\tilde{P}^{a b}}{k} \sum_{j \neq i} \frac{\mathcal{T}_{a}^{i} \mathcal{T}_{b}^{j}}{\bar{z}_{i j}}+\mathcal{O}\left(k^{-2}\right)
$$

and our result (8.1) was obtained in eq.(14.3) of [13] as the solution to this equation. The derivation of (8.1) in the present paper confirms this conjecture to the indicated order, and we observe that the conjectured form of the flat connection in (8.4) is precisely the high-level form of the flat connection $\bar{W}_{i}\left(\bar{z}, z_{0}\right)$.

Considered with the result (6.4) for the induced flat connections of the finite-level coset correlators, this observation suggests a stronger form of the conjecture, namely that

$$
\bar{W}_{i}\left(\tilde{L}, \bar{z}, z_{0}\right) \quad, \quad W_{i}\left(L, z_{0}, z\right)
$$

are the finite-level induced flat connections of the $\tilde{L}$ and the $L$ theory respectively, where $z_{0}$ is a regular reference point. This conjecture should be investigated vis-a-vis finite-level factorization, as discussed in Ref. 14.

C. $S L(2, \mathbb{R})$ covariance. With Ref. [13], we verify the expected $S L(2, \mathbb{R})$ relations,

$$
\sum_{i=1}^{n} \bar{\partial}_{i} \bar{A}\left(\bar{z}, z_{0}\right)=\sum_{i=1}^{n}\left(\bar{z}_{i} \bar{\partial}_{i}+\tilde{\Delta}_{\alpha_{i}}\right) \bar{A}\left(\bar{z}, z_{0}\right)=\sum_{i=1}^{n}\left(\bar{z}_{i}^{2} \bar{\partial}_{i}+2 \bar{z}_{i} \tilde{\Delta}_{\alpha_{i}}\right) \bar{A}\left(\bar{z}, z_{0}\right)=\mathcal{O}\left(k^{-2}\right)
$$

using the global Ward identity $(7.2 \mathrm{~b})$ and the known conformal weights $\tilde{\Delta}=$ $\operatorname{diag}\left(\tilde{L}^{a b} \mathcal{T}_{a} \mathcal{T}_{b}\right)$ of the broken affine primary fields. 
D. Two- and three-point correlators. Choosing $n=2$ in eq.(8.1), we obtain the high-level two-point correlators of ICFT,

$$
\bar{A}^{\alpha_{1} \alpha_{2}}\left(\bar{z}_{1} z_{1}^{0} \mathcal{T}^{1}, \bar{z}_{2} z_{1}^{0} \mathcal{T}^{2}\right)=\frac{\eta^{\alpha_{1} \alpha_{2}}\left(\mathcal{T}^{1}\right) \delta\left(\mathcal{T}^{2}-\overline{\mathcal{T}}^{1}\right)}{\left(z_{12}^{0}\right)^{2 \Delta_{1}^{g}}}\left(\frac{z_{12}^{0}}{\bar{z}_{12}}\right)^{2 \tilde{\Delta}_{\alpha_{1}}}+\mathcal{O}\left(k^{-2}\right)
$$

where we have used the fact that the two-point affine-Sugawara correlator $A_{g}^{\alpha_{1} \alpha_{2}}\left(z_{0}\right)=\eta^{\alpha_{1} \alpha_{2}}\left(\mathcal{T}^{1}\right) \delta\left(\mathcal{T}^{2}-\overline{\mathcal{T}}^{1}\right) /\left(z_{12}^{0}\right)^{2 \Delta_{1}^{g}}$ is proportional to the inverse carrier space metric $\eta^{\alpha_{1} \alpha_{2}}\left(\mathcal{T}^{1}\right)$ of representation $\mathcal{T}^{1}$.

Choosing $n=3$ in eq.(8.1), we obtain the high-level three-point correlators of ICFT,

$$
\begin{aligned}
& \bar{A}^{\alpha_{1} \alpha_{2} \alpha_{3}}\left(\bar{z}_{1} z_{1}^{0} \mathcal{T}^{1}, \bar{z}_{2} z_{2}^{0} \mathcal{T}^{2}, \bar{z}_{3} z_{3}^{0} \mathcal{T}^{3}\right)=A_{g}^{\alpha_{1} \alpha_{2} \alpha_{3}}\left(z_{1}^{0} \mathcal{T}^{1}, z_{2}^{0} \mathcal{T}^{2}, z_{3}^{0} \mathcal{T}^{3}\right) \\
& \times\left(\frac{z_{12}^{0}}{\bar{z}_{12}}\right)^{\tilde{\Delta}_{\alpha_{1}}+\tilde{\Delta}_{\alpha_{2}}-\tilde{\Delta}_{\alpha_{3}}}\left(\frac{z_{13}^{0}}{\bar{z}_{13}}\right)^{\tilde{\Delta}_{\alpha_{1}}+\tilde{\Delta}_{\alpha_{3}}-\tilde{\Delta}_{\alpha_{2}}}\left(\frac{z_{23}^{0}}{\bar{z}_{23}}\right)^{\tilde{\Delta}_{\alpha_{2}}+\tilde{\Delta}_{\alpha_{3}}-\tilde{\Delta}_{\alpha_{1}}}+\mathcal{O}\left(k^{-2}\right)
\end{aligned}
$$

where the three-point affine-Sugawara correlators $A_{g}^{\alpha_{1} \alpha_{2} \alpha_{3}}$ are proportional to the Clebsch-Gordan coefficients of the decomposition $\mathcal{T}^{1} \otimes \mathcal{T}^{2}$ into $\overline{\mathcal{T}}^{3}$. The result (8.8) is an independent confirmation of a conclusion reached in Ref.[14]: The high-level fusion rules of the broken affine primaries follow the Clebsch-Gordan coefficients of the representations.

E. Invariant four-point correlators. The high-level invariant four-point correlators of ICFT are known [14]. To check our results against these, recall that the invariant correlators were obtained in the $\mathrm{KZ}$ gauge, where

$$
\begin{gathered}
\bar{Y}^{\alpha}\left(\bar{u}, u_{0}\right)=\left(\prod_{i<j}^{4} \bar{z}_{i j}^{\bar{\gamma}_{i j}}\right) \bar{A}^{\alpha}\left(\bar{z}, z_{0}\right) \quad, \quad \bar{u}=\frac{\bar{z}_{12} \bar{z}_{34}}{\bar{z}_{14} \bar{z}_{32}}, \alpha=\left(\alpha_{1} \alpha_{2} \alpha_{3} \alpha_{4}\right) \\
\bar{\gamma}_{12}=\bar{\gamma}_{13}=0 \quad, \quad \bar{\gamma}_{14}=2 \tilde{\Delta}_{\alpha_{1}} \quad, \quad \bar{\gamma}_{23}=\tilde{\Delta}_{\alpha_{1}}+\tilde{\Delta}_{\alpha_{2}}+\tilde{\Delta}_{\alpha_{3}}-\tilde{\Delta}_{\alpha_{4}} \\
\bar{\gamma}_{24}=-\tilde{\Delta}_{\alpha_{1}}+\tilde{\Delta}_{\alpha_{2}}-\tilde{\Delta}_{\alpha_{3}}+\tilde{\Delta}_{\alpha_{4}} \quad, \quad \bar{\gamma}_{34}=-\tilde{\Delta}_{\alpha_{1}}-\tilde{\Delta}_{\alpha_{2}}+\tilde{\Delta}_{\alpha_{3}}+\tilde{\Delta}_{\alpha_{4}}
\end{gathered}
$$

Substituting our result (8.1a) for $n=4$ into (8.9a), we find

$$
\bar{Y}^{\alpha}\left(\bar{u}, u_{0}\right)=Y_{\tilde{L}}^{\alpha}\left(\bar{u}, u_{0}\right) d(\alpha) \quad, \quad d(\alpha) \equiv \prod_{i<j}^{4}\left(z_{i j}^{0}\right)^{\bar{\gamma}_{i j}}
$$


after some algebra with the global Ward identity (7.2b). Here, $d(\alpha)$ is an irrelevant constant and the $\bar{u}$-dependent factor,

$$
\begin{gathered}
Y_{\tilde{L}}^{\alpha}\left(\bar{u}, u_{0}\right)=Y_{g}^{\beta}\left(u_{0}\right)\left(\mathbb{1}+\frac{\tilde{P}_{a b}}{k}\left[\mathcal{T}_{a}^{1} \mathcal{T}_{b}^{2} \ln \left(\frac{\bar{u}}{u_{0}}\right)+\mathcal{T}_{a}^{1} \mathcal{T}_{b}^{3} \ln \left(\frac{1-\bar{u}}{1-u_{0}}\right)\right]\right)_{\beta}{ }^{\alpha}+\mathcal{O}\left(k^{-2}\right) \\
Y_{g}\left(u_{0}\right)=A_{g}\left(u_{0}\right) \quad, \quad \tilde{L}^{a b}=\frac{\tilde{P}^{a b}}{2 k}+\mathcal{O}\left(k^{-2}\right)
\end{gathered}
$$

is exactly the result of Ref. [14.

F. Induced non-local conserved quantities. As seen for the coset correlators in Section 6, the non-local conserved quantities of the high-level biconformal correlators induce non-local conserved quantities for the high-level conformal correlators.

To see this, we need the induction identity

$$
\begin{aligned}
\left(\mathbb{1}+\frac{P^{a b}}{k} \sum_{i<j} \mathcal{T}_{a}^{i} \mathcal{T}_{b}^{j}\right. & \left.\ln \left(\frac{z_{i j}}{z_{i j}^{0}}\right)\right) Q_{a}(\bar{z}, z) \\
& =Q_{a}\left(\bar{z}, z_{0}\right)\left(1+\frac{P^{a b}}{k} \sum_{i<j} \mathcal{T}_{a}^{i} \mathcal{T}_{b}^{j} \ln \left(\frac{z_{i j}}{z_{i j}^{0}}\right)\right)+\mathcal{O}\left(k^{-2}\right)
\end{aligned}
$$

which follows from (7.1d) in analogy with eq.(6.5). Then, following the argument in (6.6) and (6.7), we find that

$$
\begin{gathered}
\bar{A}\left(\bar{z}, z_{0}\right) Q_{a}\left(\bar{z}, z_{0}\right)=\mathcal{O}\left(k^{-2}\right) \quad, \quad a=1, \ldots, \operatorname{dim} g \\
Q_{a}\left(\bar{z}, z_{0}\right)=Q_{a}^{g}+\left[Q_{a}^{g}, \frac{\tilde{P}^{a b}}{k} \sum_{i<j} \mathcal{T}_{a}^{i} \mathcal{T}_{b}^{j} \ln \left(\frac{\bar{z}_{i j}}{z_{i j}^{0}}\right)\right]+\mathcal{O}\left(k^{-2}\right) \\
=\sum_{i=1}^{n} \mathcal{T}_{a}^{i}+\frac{i \tilde{P}^{b(c} f_{a b}{ }^{d)}}{k} \sum_{i<j} \mathcal{T}_{c}^{i} \mathcal{T}_{d}^{j} \ln \left(\frac{\bar{z}_{i j}}{z_{i j}^{0}}\right)+\mathcal{O}\left(k^{-2}\right)
\end{gathered}
$$

is induced from the invariance (7.1c) of the biconformal correlators. This result can also be verified directly from the high-level correlators in (8.1).

Taken together with the induced non-local $g$-invariance (6.7) of the finitelevel coset correlators, the result (8.13) strongly suggests that

$$
Q_{a}\left(\bar{z}, z_{0}\right) \quad, \quad Q_{a}\left(z_{0}, z\right)
$$


are the finite-level induced non-local conserved quantities for the $\tilde{L}$ and $L$ theory respectively. This conjecture should be considered in parallel with the conjectured form of the induced flat connections in eq.(8.5).

G. Physical singularities. The high-level correlators (8.1) exhibit the correct singularities in all channels $\bar{z}_{i} \rightarrow \bar{z}_{j}$. For simplicity, we discuss this result for $\bar{z}_{1} \rightarrow \bar{z}_{2}$, called the 1-2 channel, but any pair of $\bar{z}$ 's may be similarly chosen.

To begin, we expand the affine-Sugawara correlator $A_{g}^{\alpha}\left(z_{0}\right)$ in (8.1a) in a complete set of invariant tensors $v_{n}^{\alpha}$ of $\mathcal{T}^{1} \otimes \cdots \otimes \mathcal{T}^{n}$,

$$
\begin{gathered}
A_{g}^{\alpha}\left(z_{0}\right)=\sum_{r, \xi,[R]} \mathcal{A}_{g}^{(n)}\left(r, \xi,[R] ; z_{0}\right) v_{n}^{\alpha}(r, \xi,[R]) \\
A_{g}\left(z_{0}\right) Q_{a}^{g}=v_{n}(r, \xi,[R]) Q_{a}^{g}=0 \quad, \quad Q_{a}^{g}=\sum_{i=1}^{n} \mathcal{T}_{a}^{i}
\end{gathered}
$$

where the coefficients $\mathcal{A}_{g}^{(n)}$ are related to the $n$-point affine-Sugawara blocks at $z_{0}$. In (8.15), we have chosen the invariant tensors in a basis appropriate to the 1-2 channel,

$$
\begin{gathered}
v_{n}^{\alpha_{1} \alpha_{2} \ldots \alpha_{n}}(r, \xi,[R])=\sum_{\alpha_{r} \alpha_{\bar{\tau}}} v_{3}^{\alpha_{1} \alpha_{2} \alpha_{r}}(r, \xi) \eta_{\alpha_{r} \alpha_{\bar{r}}}\left(\mathcal{T}^{r}\right) v_{n-1}^{\alpha_{\bar{\tau}} \alpha_{3} \ldots \alpha_{n}}([R]) \\
v_{3}\left(\mathcal{T}_{a}^{1}+\mathcal{T}_{a}^{2}+\mathcal{T}_{a}^{r}\right)=0 \quad, \quad v_{n-1}\left(\overline{\mathcal{T}}_{a}^{r}+\mathcal{T}_{a}^{3}+\ldots+\mathcal{T}_{a}^{n}\right)=0
\end{gathered}
$$

where $\mathcal{T}^{r}$ is an irrep of $g$ in the 1-2 channel, $\eta_{\alpha_{r} \alpha_{\bar{r}}}\left(\mathcal{T}^{r}\right)$ is its carrier space metric, and $v_{3}^{\alpha}, v_{n-1}^{\alpha}$ are the invariant tensors of

$$
\mathcal{T}^{1} \otimes \mathcal{T}^{2} \otimes \mathcal{T}^{r} \quad, \quad \overline{\mathcal{T}}^{r} \otimes \mathcal{T}^{3} \otimes \cdots \otimes \mathcal{T}^{n}
$$

respectively. The argument $\xi$ in (8.15), (8.16a) labels different copies of $\mathcal{T}^{r}$ and $[R]$ collects the other couplings of $v_{n-1}$. The result (8.16) was obtained by studying Haar integration over $n$ matrix representations $g\left(\mathcal{T}^{i}\right), i=1, \ldots, n$ of the group $g$.

It will also be helpful to recall that [14]

$$
\begin{gathered}
v_{3}^{\alpha_{i} \alpha_{j} \alpha_{r}}(r, \xi)=\sum_{\alpha_{\bar{r}}}\left(\begin{array}{ccc}
\alpha_{i} & \alpha_{j} & \bar{r}(\xi) \\
i & j & \alpha_{\bar{r}}
\end{array}\right) \eta^{\alpha_{\bar{r}} \alpha_{r}}\left(\mathcal{T}^{r}\right) \\
v_{3}^{\beta_{1} \beta_{2} \alpha_{r}}(r, \xi)\left[2 \tilde{L}^{a b} \mathcal{T}_{a}^{1} \mathcal{T}_{b}^{2}\right]_{\beta_{1} \beta_{2}}{ }^{\alpha_{1} \alpha_{2}}=v_{3}^{\alpha_{1} \alpha_{2} \alpha_{r}}(r, \xi)\left(\tilde{\Delta}_{\alpha_{r}}\left(\mathcal{T}^{r}\right)-\tilde{\Delta}_{\alpha_{1}}\left(\mathcal{T}^{1}\right)-\tilde{\Delta}_{\alpha_{2}}\left(\mathcal{T}^{2}\right)\right)
\end{gathered}
$$


where $(\cdots)$ is the Clebsch-Gordan coefficient of $\mathcal{T}^{i} \otimes \mathcal{T}^{j}$ into $\overline{\mathcal{T}}^{r}$.

Using (8.15), (8.16) and (8.18b), we extract the form of the high-level correlators as $\bar{z}_{1} \rightarrow \bar{z}_{2}$,

$$
\begin{gathered}
\bar{A}^{\alpha}\left(\bar{z}, z_{0}\right) \underset{\bar{z}_{1} \rightarrow \bar{z}_{2}}{\simeq} \sum_{\substack{r, \xi,[R] \\
\alpha_{r} \alpha_{\bar{r}}}} \mathcal{A}_{g}\left(r, \xi,[R] ; z_{0}\right) v_{3}^{\alpha_{1} \alpha_{2} \alpha_{r}}(r, \xi) \eta_{\alpha_{r} \alpha_{\bar{r}}}\left(\mathcal{T}^{r}\right)\left(\frac{\bar{z}_{12}}{z_{12}^{0}}\right)^{\tilde{\Delta}_{\alpha_{r}}-\tilde{\Delta}_{\alpha_{1}}-\tilde{\Delta}_{\alpha_{2}}} \\
\quad \times A_{(n-1)}^{\alpha_{\bar{r}} \alpha_{3} \ldots \alpha_{n}}\left(\bar{z}_{2} z_{2}^{0} \overline{\mathcal{T}}^{r}, \bar{z}_{3} z_{3}^{0} \mathcal{T}^{3}, \ldots, \bar{z}_{n} z_{n}^{0} \mathcal{T}^{n}\right)[R]+\mathcal{O}\left(k^{-2}\right) \\
A_{(n-1)}^{\alpha_{\bar{r}} \alpha_{3} \ldots \alpha_{n}}\left(\bar{z}_{2} z_{2}^{0} \overline{\mathcal{T}}^{r}, \bar{z}_{3} z_{3}^{0} \mathcal{T}^{3}, \ldots, \bar{z}_{n} z_{n}^{0} \mathcal{T}^{n}\right)[R] \\
=v_{n-1}^{\beta_{\bar{r}} \beta_{3} \ldots \beta_{n}}([R])\left[1+\frac{\tilde{P}^{a b}}{k}\left(\sum_{j=3}^{n} \overline{\mathcal{T}}_{a}^{r} \mathcal{T}_{b}^{j} \ln \left(\frac{\bar{z}_{2 j}}{z_{2 j}^{0}}\right)+\sum_{3 \leq i<j}^{n} \mathcal{T}_{a}^{i} \mathcal{T}_{b}^{j} \ln \left(\frac{\bar{z}_{i j}}{z_{i j}^{0}}\right)\right)\right]_{\beta_{\bar{r}} \beta_{3} \ldots \beta_{n}}^{\alpha_{\bar{n}} \alpha_{3} \ldots \alpha_{n}} .
\end{gathered}
$$

This result shows the correct factorization of the high-level $n$-point correlators into the high-level three-point correlators times the high-level $(n-1)$-point correlators, including the correct conformal weight factor for broken affine primaries in the 1-2 channel. Since the three-point invariant tensors (8.18a) are proportional to the Clebsch-Gordan coefficients, the high-level fusion rules [14] of ICFT are also visible here.

\section{Conclusions}

Irrational conformal field theory (ICFT) includes rational conformal field theory as a small subspace, and the affine-Virasoro Ward identities describe the biconformal correlators of ICFT.

We have seen that the Ward identities are equivalent to a linear partial differential system with flat connections and unsuspected new non-local conserved quantities. In this formulation, the equations of ICFT are clearly seen as generalized $\mathrm{KZ}$ equations, including the $\mathrm{KZ}$ equation itself as the simplest example.

As examples of the new formulation we solved for the coset correlators, the correlators of the affine-Sugawara nests and the high-level $n$-point correlators of ICFT. In the latter case, our results agree with the known high-level four-point correlators and continue to show good physical behavior for $n \neq 4$. 
Having seen this elegant structure in ICFT, we may remark on an outstanding technical problem of the program: We have been studying ICFT primarily by pedestrian methods, that is, by computing and summing the connection moments. It is important now to develop new methods which compute the flat connections more directly.

\section{Acknowledgments}

We thank M. Douglas for suggesting this investigation.

We also thank the Niels Bohr Institute and the CERN Theory Division for their hospitality during part of this work. NO also thanks the Theoretical High Energy Physics Group at Nijmegen University for their hospitality.

The work of $\mathrm{MBH}$ was supported in part by the Director, Office of Energy Research, Office of High Energy and Nuclear Physics, Division of High Energy Physics of the U.S. Department of Energy under Contract DE-AC03-76SF00098 and in part by the National Science Foundation under grant PHY90-21139. The work of NO was supported in part by a Séjour Scientifique de Longue Durée of the Ministère des Affaires Etrangères.

\section{Appendix A: Invariant flat connections}

Following the development in Sections 2 and 3, we may also translate the invariant four-point Ward identities $[13,14]$

$$
\begin{gathered}
\left.\bar{\partial}^{q} \partial^{p} Y(\bar{u}, u)\right|_{\bar{u}=u}=Y_{g}(u) W_{q p}(u) \\
Y_{g}(u)=Y(u, u) \quad, \quad Y_{g}(u) Q_{a}^{g}=0 \quad, \quad Q_{a}^{g}=\sum_{i=1}^{4} \mathcal{T}_{a}^{i}
\end{gathered}
$$

into the system of invariant flat connections,

$$
\begin{gathered}
\bar{\partial} Y(\bar{u}, u)=Y(\bar{u}, u) \bar{W}(\bar{u}, u) \quad, \quad \partial Y(\bar{u}, u)=Y(\bar{u}, u) W(\bar{u}, u) \\
Y(\bar{u}, u) Q_{a}(\bar{u}, u)=0 \quad, \quad a=1, \ldots, \operatorname{dim} g
\end{gathered}
$$

where $Y_{g}(u)$ is the invariant affine-Sugawara correlator, $Y(\bar{u}, u)$ is the invariant four-point biconformal correlator and $Q_{a}(\bar{u}, u)$ are the invariant non-local conserved generators of Lie $g$. 
Drawing on known results $[13,14]$ for the invariant system, we obtain the following partial list of relevant relations,

$$
\begin{aligned}
& (\partial+W) \bar{W}=(\bar{\partial}+\bar{W}) W \\
& W_{q p}=\left.(\bar{\partial}+\bar{W})^{q}(\partial+W)^{p} \mathbb{1}\right|_{\bar{u}=u} \\
& Y(\bar{u}, u)=Y_{g}(u) \bar{F}(\bar{u}, u)=Y_{g}(\bar{u}) F(\bar{u}, u) \\
& Y_{g}(u)=Y_{g}\left(u_{0}\right) Y\left(u, u_{0}\right) \quad, \quad \partial_{u} Y_{g}\left(u, u_{0}\right)=Y_{g}\left(u, u_{0}\right) W^{g}(u) \quad, \quad Y_{g}\left(u_{0}, u_{0}\right)=\mathbb{1} \\
& \bar{F}(\bar{u}, u) \equiv U^{*} \mathrm{e}^{\int_{u}^{\bar{u}} \mathrm{~d} \bar{u}^{\prime} \bar{W}\left(\bar{u}^{\prime}, u\right)}=\sum_{q=0}^{\infty} \frac{(\bar{u}-u)^{q}}{q !} W_{q 0}(u) \\
& F(\bar{u}, u) \equiv U^{*} \mathrm{e}^{\int_{\bar{u}}^{u} \mathrm{~d} u^{\prime} W\left(\bar{u}, u^{\prime}\right)}=\sum_{p=0}^{\infty} \frac{(u-\bar{u})^{p}}{p !} W_{0 p}(\bar{u}) \\
& \bar{F}(\bar{u}, u)=Y_{g}(\bar{u}, u) F(\bar{u}, u) \\
& \bar{W}=\bar{F}^{-1} \bar{\partial} \bar{F}=F^{-1}\left(\bar{\partial}+W^{g}(\bar{u})\right) F \\
& W=F^{-1} \partial F=\bar{F}^{-1}\left(\partial+W^{g}(u)\right) \bar{F} \\
& \bar{W}(u, u)=W_{10}(u)=2 \tilde{L}^{a b}\left(\frac{\mathcal{T}_{a}^{1} \mathcal{T}_{b}^{2}}{u}+\frac{\mathcal{T}_{a}^{1} \mathcal{T}_{b}^{3}}{u-1}\right) \\
& W(u, u)=W_{01}(u)=2 L^{a b}\left(\frac{\mathcal{T}_{a}^{1} \mathcal{T}_{b}^{2}}{u}+\frac{\mathcal{T}_{a}^{1} \mathcal{T}_{b}^{3}}{u-1}\right) \\
& \bar{W}(u, u)+W(u, u)=W^{g}(u)=2 L_{g}^{a b}\left(\frac{\mathcal{T}_{a}^{1} \mathcal{T}_{b}^{2}}{u}+\frac{\mathcal{T}_{a}^{1} \mathcal{T}_{b}^{3}}{u-1}\right) \\
& Q_{a}(\bar{u}, u)=F^{-1}(\bar{u}, u) Q_{a}^{g} F(\bar{u}, u)=\bar{F}^{-1}(\bar{u}, u) Q_{a}^{g} \bar{F}(\bar{u}, u) \\
& {\left[Q_{a}(\bar{u}, u), Q_{b}(\bar{u}, u)\right]=i f_{a b}{ }^{c} Q_{c}(\bar{u}, u)} \\
& \bar{D} Q_{a} \equiv \bar{\partial} Q_{a}+\left[\bar{W}, Q_{a}\right]=0 \quad, \quad D Q_{a} \equiv \partial Q_{a}+\left[W, Q_{a}\right]=0 \\
& \bar{D}\left(Y Q_{a}\right)=\bar{\partial}\left(Y Q_{a}\right)-\left(Y Q_{a}\right) \bar{W}=0 \quad, \quad D\left(Y Q_{a}\right)=\partial\left(Y Q_{a}\right)-\left(Y Q_{a}\right) W=0 \\
& Y_{g}(u) Q_{a}^{g}=0 \leftrightarrow Y\left(\bar{u}_{0}, u_{0}\right) Q_{a}\left(\bar{u}_{0}, u_{0}\right)=0 \leftrightarrow Y(\bar{u}, u) Q_{a}(\bar{u}, u)=0
\end{aligned}
$$

in complete analogy with the results of Section 2 and 3. In (A.3e) and (A.3f), $U^{*}$ is anti-ordering in $u$. 
For K-conjugation and crossing symmetry we obtain the relations,

$$
\begin{array}{rrr}
\bar{F}(\tilde{L}, \bar{u}, u) & , \quad F(L, \bar{u}, u) & (A .4 a) \\
\bar{W}(\tilde{L}, \bar{u}, u) & , \quad W(L, \bar{u}, u) & (A .4 b) \\
\bar{F}\left(\tilde{L}=L_{*}, \bar{u}, u\right)=F\left(L=L_{*}, u, \bar{u}\right) & , \quad F\left(L=L_{*}, \bar{u}, u\right)=\bar{F}\left(\tilde{L}=L_{*}, u, \bar{u}\right) \\
\bar{W}\left(\tilde{L}=L_{*}, \bar{u}, u\right)=W\left(L=L_{*}, u, \bar{u}\right) & , \quad W\left(L=L_{*}, \bar{u}, u\right)=\bar{W}\left(\tilde{L}=L_{*}, u, \bar{u}\right) \\
\left.Y(\bar{u}, u)\right|_{\tilde{L} \leftrightarrow L}=Y(u, \bar{u}) & (A .4 d) \\
\left.Q_{a}(\bar{u}, u)\right|_{\tilde{L} \leftrightarrow L}=Q_{a}(u, \bar{u}) & (A .4 e) \\
\bar{F}(1-\bar{u}, 1-u)=P_{23} \bar{F}(\bar{u}, u) P_{23} \quad, \quad F(1-\bar{u}, 1-u)=P_{23} F(\bar{u}, u) P_{23} & (A .4 g \\
\bar{W}(1-\bar{u}, 1-u)=-P_{23} \bar{W}(\bar{u}, u) P_{23} & , \quad W(1-\bar{u}, 1-u)=-P_{23} W(\bar{u}, u) P_{23} \\
Q_{a}(1-\bar{u}, 1-u)=P_{23} Q_{a}(\bar{u}, u) P_{23} & (A .4 h) \\
\end{array}
$$

where $P_{23}$ is the exchange operator which satisfies $P_{23} \mathcal{T}^{2} P_{23}=\mathcal{T}^{3}, P_{23}^{2}=1$.

For the general nest, we find the invariant evolution operators,

$$
\begin{array}{r}
\bar{F}\left[L=L_{g / h_{1} / \ldots / h_{2 n+1}}, \bar{u}, u\right]=Y_{g}(\bar{u}, u) Y_{h_{1}}(u, \bar{u}) \cdots Y_{h_{2 n+1}}(u, \bar{u}) \\
F\left[L=L_{h_{1} / \ldots / h_{2 n+1}}, \bar{u}, u\right]=Y_{h_{1}}(u, \bar{u}) \cdots Y_{h_{2 n+1}}(u, \bar{u}) \\
\bar{F}\left[L=L_{g / h_{1} / \ldots / h_{2 n}}, \bar{u}, u\right]=Y_{g}(\bar{u}, u) Y_{h_{1}}(u, \bar{u}) \cdots Y_{h_{2 n}}(\bar{u}, u) \\
F\left[L=L_{h_{1} / \ldots / h_{2 n}}, \bar{u}, u\right]=Y_{h_{1}}(u, \bar{u}) \cdots Y_{h_{2 n}}(\bar{u}, u)
\end{array}
$$

where $Y_{h_{i}}\left(u, u_{0}\right)$ is the invariant evolution operator of $h_{i}$,

$$
\partial Y_{h_{i}}\left(u, u_{0}\right)=Y_{h_{i}}\left(u, u_{0}\right) W^{h_{i}}(u) \quad, \quad Y_{h_{i}}\left(u_{0}, u_{0}\right)=\mathbb{1}
$$

Using (A.3c,h,i) and (A.5), one may obtain the invariant flat connections $\bar{W}, W$ of the nests and their known [14] invariant four-point biconformal correlators. We have also checked that $\bar{W}\left(\bar{u}, u_{0}\right)$ and $Q_{a}\left(\bar{u}, u_{0}\right)$ are the induced connections and induced non-local conserved generators of $g$ for the invariant coset correlators $\left(Y_{g}\left(u_{0}\right) Y_{h}^{-1}\left(u, u_{0}\right)\right)^{\alpha}$ obtained in Ref. 13. 
The relevant high-level results are:

$$
\begin{aligned}
& \bar{F}(\bar{u}, u)=\mathbb{1}+\frac{\tilde{P}^{a b}}{k}\left(\mathcal{T}_{a}^{1} \mathcal{T}_{b}^{2} \ln \left(\frac{\bar{u}}{u}\right)+\mathcal{T}_{a}^{1} \mathcal{T}_{b}^{3} \ln \left(\frac{1-\bar{u}}{1-u}\right)\right)+\mathcal{O}\left(k^{-2}\right) \\
& F(\bar{u}, u)=\mathbb{1}+\frac{P^{a b}}{k}\left(\mathcal{T}_{a}^{1} \mathcal{T}_{b}^{2} \ln \left(\frac{u}{\bar{u}}\right)+\mathcal{T}_{a}^{1} \mathcal{T}_{b}^{3} \ln \left(\frac{1-u}{1-\bar{u}}\right)\right)+\mathcal{O}\left(k^{-2}\right) \\
& \bar{W}(\bar{u}, u)=W_{10}(\bar{u})+\mathcal{O}\left(k^{-2}\right) \\
& W(\bar{u}, u)=W_{01}(u)+\mathcal{O}\left(k^{-2}\right) \\
& Y(\bar{u}, u)=Y_{g}\left(u_{0}\right)\left[\mathbb{1}+\frac{\tilde{P}^{a b}}{k}\left(\mathcal{T}_{a}^{1} \mathcal{T}_{b}^{2} \ln \left(\frac{\bar{u}}{u_{0}}\right)+\mathcal{T}_{a}^{1} \mathcal{T}_{b}^{3} \ln \left(\frac{1-\bar{u}}{1-u_{0}}\right)\right)\right] \\
& \times\left[\mathbb{1}+\frac{P^{a b}}{k}\left(\mathcal{T}_{a}^{1} \mathcal{T}_{b}^{2} \ln \left(\frac{u}{u_{0}}\right)+\mathcal{T}_{a}^{1} \mathcal{T}_{b}^{3} \ln \left(\frac{1-u}{1-u_{0}}\right)\right)\right]+\mathcal{O}\left(k^{-2}\right) \\
& Y(\bar{u}, u) Q_{a}^{g}(\bar{u}, u)=\mathcal{O}\left(k^{-2}\right) \\
& Q_{a}(\bar{u}, u)=Q_{a}^{g}+\left[Q_{a}^{g}, \frac{P^{a b}}{k}\left(\mathcal{T}_{a}^{1} \mathcal{T}_{a}^{2} \ln \left(\frac{u}{\bar{u}}\right)+\mathcal{T}_{a}^{1} \mathcal{T}_{b}^{3} \ln \left(\frac{1-u}{1-\bar{u}}\right)\right)\right]+\mathcal{O}\left(k^{-2}\right) \\
& =Q_{a}^{g}+\left[Q_{a}^{g}, \frac{\tilde{P}^{a b}}{k}\left(\mathcal{T}_{a}^{1} \mathcal{T}_{a}^{2} \ln \left(\frac{\bar{u}}{u}\right)+\mathcal{T}_{a}^{1} \mathcal{T}_{b}^{3} \ln \left(\frac{1-\bar{u}}{1-u}\right)\right)\right]+\mathcal{O}\left(k^{-2}\right) \\
& Y_{\tilde{L}}\left(\bar{u}, u_{0}\right) Q_{a}^{g}\left(\bar{u}, u_{0}\right)=\mathcal{O}\left(k^{-2}\right) \quad .
\end{aligned}
$$

The high-level invariant conformal correlators $Y_{\tilde{L}}\left(\bar{u}, u_{0}\right)$ in $(8.11 \mathrm{a})$ and $(\mathrm{A} .7 \mathrm{i})$ can be read from the factorized form of the high-level invariant biconformal correlators in (A.7e). We have also checked that $\bar{W}\left(\bar{u}, u_{0}\right)$ and $Q_{a}\left(\bar{u}, u_{0}\right)$ are the induced connection and induced non-local conserved generators of $g$ for the high-level correlators $Y_{\tilde{L}}\left(\bar{u}, u_{0}\right)$.

\section{Appendix B: Flat connections and connection moments}

In this appendix, we work out in further detail the formula of the text,

$$
\begin{gathered}
W_{i}(\bar{z}, z)=F^{-1}(\bar{z}, z) \partial_{i} F(\bar{z}, z) \\
F(\bar{z}, z)=\sum_{p=0}^{\infty} \frac{1}{p !} \sum_{i_{1} \ldots i_{p}} \prod_{\mu=1}^{p}\left(z_{i_{\mu}}-\bar{z}_{i_{\mu}}\right) W_{0, i_{1} \ldots i_{p}}(\bar{z})
\end{gathered}
$$


which expresses the flat connection $W_{i}$ in terms of its one-sided connection moments $W_{0, i_{1} \ldots i_{p}}$.

Using $(1+x)^{-1}=\sum_{r=0}^{\infty}(-)^{r} x^{r}$, we have the more explicit form of the flat connection,

$$
\begin{aligned}
W_{i}(\bar{z}, z)= & {\left[\sum_{r=0}^{\infty}(-)^{r}\left(\sum_{p=1}^{\infty} \frac{1}{p !} \sum_{i_{1} \ldots i_{p}} \prod_{\mu=1}^{p}\left(z_{i_{\mu}}-\bar{z}_{i_{\mu}}\right) W_{0, i_{1} \ldots i_{p}}(\bar{z})\right)^{r}\right] } \\
& \times\left(\sum_{s=0}^{\infty} \frac{1}{s !} \sum_{k_{1} \ldots k_{s}} \prod_{\nu=1}^{s}\left(z_{k_{\nu}}-\bar{z}_{k_{\nu}}\right) W_{0, k_{1} \ldots k_{s} i}(\bar{z})\right)
\end{aligned}
$$

which can be rearranged as follows,

$$
\begin{gathered}
W_{i}(\bar{z}, z)=\sum_{p=0}^{\infty} \frac{1}{p !} \sum_{i_{1} \ldots i_{p}} \prod_{\mu=1}^{p}\left(z_{i_{\mu}}-\bar{z}_{i_{\mu}}\right) \rho_{i_{1} \ldots i_{p} i}(\bar{z}) \\
\left.\rho_{i_{1} \ldots i_{p} i}(\bar{z}) \equiv \partial_{i_{1}} \cdots \partial_{i_{p}} W_{i}(\bar{z}, z)\right|_{z=\bar{z}} \\
=\sum_{r=0}^{\infty}(-)^{r} \sum_{p_{1}=1}^{\infty} \cdots \sum_{p_{r}=1}^{\infty} \sum_{s=0}^{\infty} \delta\left(p-s-\sum_{i=1}^{r} p_{i}\right) \frac{1}{s ! \prod_{i=1}^{r} p_{i} !} \\
\times \sum_{\mathcal{P}\left(i_{1} \ldots i_{p}\right)} W_{0, i_{1} \ldots i_{p_{1}}} W_{0, i_{p_{1}+1} \ldots i_{p_{1}+p_{2}}} \cdots W_{0, i_{p-p_{r}-s+1 \ldots i_{p-s}} W_{0, i_{p-s+1} \ldots i_{p} i}} .
\end{gathered}
$$

This is a computationally convenient form of the flat connection, and we find, for example, that

$$
\begin{gathered}
\rho_{i}=W_{0, i} \\
\rho_{i_{1} i}=W_{0, i_{1} i}-W_{0, i_{1}} W_{0, i} \\
\rho_{i_{1} i_{2} i}=W_{0, i_{1} i_{2} i}-W_{0, i_{1}} W_{\left.0, i_{2}\right) i}-W_{0, i_{1} i_{2}} W_{0, i}+W_{0, i_{1}} W_{\left.0, i_{2}\right)} W_{0, i} \\
=\left[\frac{1}{2} W_{0, i_{1} i_{2} i}-W_{0, i_{1}} W_{0, i_{2} i}-\frac{1}{2} W_{0, i_{1} i_{2}} W_{0, i}+W_{0, i_{1}} W_{0, i_{2}} W_{0, i}\right]+\left(i_{1} \leftrightarrow i_{2}\right)
\end{gathered} .
$$

Similarly, we have the alternate expansion,

$$
\begin{aligned}
W_{i}(\bar{z}, z) & =\sum_{p=0}^{\infty} \frac{1}{p !} \sum_{i_{1} \ldots i_{p}} \prod_{\mu=1}^{p}\left(\bar{z}_{i_{\mu}}-z_{i_{\mu}}\right) \sigma_{i_{1} \ldots i_{p} i}(z) \\
\sigma_{i_{1} \ldots i_{p} i}(z) & \left.\equiv \bar{\partial}_{i_{1}} \cdots \bar{\partial}_{i_{p}} W_{i}(\bar{z}, z)\right|_{\bar{z}=z} \\
& =\sum_{r=0}^{p} \frac{(-)^{p-r}}{(p-r) ! r !} \sum_{\mathcal{P}\left(i_{1} \ldots i_{p}\right)} \partial_{i_{1}} \cdots \partial_{i_{r}} \rho_{i_{r+1} \ldots i_{p} i}(z)
\end{aligned}
$$




$$
\begin{gathered}
\sigma_{i}=W_{0, i} \\
\sigma_{i_{1} i}=\partial_{i_{1}} W_{0, i}-W_{0, i_{1}}+W_{0, i_{1}} W_{0, i} \\
\sigma_{i_{1} i_{2} i}=\partial_{i_{1}} \partial_{i_{2}} W_{0, i}+\partial_{\left(i_{1}\right.} W_{\left.0, i_{2}\right)} W_{0, i}-\partial_{\left(i_{1}\right.} W_{\left.0, i_{2}\right) i}+W_{0, i_{i} i_{2} i} \\
-W_{0, i_{1}} W_{\left.0, i_{2}\right) i}-W_{0, i_{1} i_{2}} W_{0, i}+W_{0, i_{1}} W_{\left.0, i_{2}\right)} W_{0, i}
\end{gathered} .
$$

Using (2.17a), similar results for $\bar{W}_{i}=\bar{F}^{-1} \bar{\partial}_{i} \bar{F}$ are easily obtained.

Using eqs.(A.3f) and (A.3i) of Appendix A, we also give the corresponding results for the invariant flat connection and its one-sided connection moments:

$$
\begin{gathered}
W(\bar{u}, u)=\left[\sum_{r=0}^{\infty}(-)^{r}\left(\sum_{p=1}^{\infty} \frac{(u-\bar{u})^{p}}{p !} W_{0 p}(\bar{u})\right)^{r}\right]\left(\sum_{s=0}^{\infty} \frac{(u-\bar{u})^{s}}{s !} W_{0, s+1}(\bar{u})\right) \\
=\sum_{p=0}^{\infty} \frac{(u-\bar{u})^{p}}{p !} \rho_{p}(\bar{u})=\sum_{p=0}^{\infty} \frac{(\bar{u}-u)^{p}}{p !} \sigma_{p}(u) \\
\left.\rho_{p}(\bar{u}) \equiv \partial^{p} W(\bar{u}, u)\right|_{u=\bar{u}} \quad,\left.\quad \sigma_{p}(u) \equiv \bar{\partial}^{p} W(\bar{u}, u)\right|_{\bar{u}=u}=\sum_{r=0}^{p}(-)^{p-r}\left(\begin{array}{l}
p \\
r
\end{array}\right) \partial^{r} \rho_{p-r}(u) \\
\rho_{p}(\bar{u})=\sum_{r=0}^{\infty}(-)^{r} \sum_{p_{1}=1}^{\infty} \cdots \sum_{p_{r}=1}^{\infty} \sum_{s=0}^{\infty} \delta\left(p-s-\sum_{i=1}^{r} p_{i}\right) \frac{p !}{s ! \prod_{i=1}^{r} p_{i} !} W_{0 p_{1}} W_{0 p_{2}} \cdots W_{0 p_{r}} W_{0, s+1} \\
\rho_{0}=W_{01}
\end{gathered}
$$

\section{Appendix C: Non-local generators of $\mathrm{g}$ and connection moments}

In this appendix, we work out in further detail the formula of the text,

$$
Q_{a}(\bar{z}, z)=F^{-1}(\bar{z}, z) Q_{a}^{g} F(\bar{z}, z) \quad, \quad a=1, \ldots, \operatorname{dim} g
$$


which expresses the non-local generators of $g$ in terms of the global generators of $g$ and the one-sided connection moments $W_{o, i_{1} \ldots i_{p}}$.

Following the development of Appendix B, we find

$$
\begin{aligned}
& Q_{a}(\bar{z}, z)=\sum_{p=0}^{\infty} \frac{1}{p !} \sum_{i_{1} \ldots i_{p}} \prod_{\mu=1}^{p}\left(z_{i_{\mu}}-\bar{z}_{i_{\mu}}\right)\left(q_{a}\right)_{i_{1} \ldots i_{p}}(\bar{z}) \\
& =\sum_{p=0}^{\infty} \frac{1}{p !} \sum_{i_{1} \ldots i_{p}} \prod_{\mu=1}^{p}\left(\bar{z}_{i_{\mu}}-z_{i_{\mu}}\right)\left(\bar{q}_{a}\right)_{i_{1} \ldots i_{p}}(z) \\
& \left.\left(q_{a}\right)_{i_{1} \ldots i_{p}}(\bar{z}) \equiv \partial_{i_{1}} \cdots \partial_{i_{p}} Q_{a}(\bar{z}, z)\right|_{z=\bar{z}} \quad,\left.\quad\left(\bar{q}_{a}\right)_{i_{1} \ldots i_{p}}(z) \equiv \bar{\partial}_{i_{1}} \cdots \bar{\partial}_{i_{p}} Q_{a}(\bar{z}, z)\right|_{\bar{z}=z} \\
& \left(q_{a}\right)_{i_{1} \ldots i_{p}}(\bar{z})=\sum_{r=0}^{\infty}(-)^{r} \sum_{p_{1}=1}^{\infty} \cdots \sum_{p_{r}=1}^{\infty} \sum_{s=0}^{\infty} \delta\left(p-s-\sum_{i=1}^{r} p_{i}\right) \frac{1}{s ! \prod_{i=1}^{r} p_{i} !} \\
& \times \sum_{\mathcal{P}\left(i_{1} \ldots i_{p}\right)} W_{0, i_{1} \ldots i_{p_{1}}} W_{0, i_{p_{1}+1} \ldots i_{p_{1}+p_{2}}} \cdots W_{0, i_{p-p_{r}-s+1} \ldots i_{p-s}} Q_{a}^{g} W_{0, i_{p-s+1} \ldots i_{p}} \\
& \left(q_{a}\right)=Q_{a}^{g} \\
& \left(q_{a}\right)_{i_{1}}=\left[Q_{a}^{g}, W_{0, i_{1}}\right] \\
& \left(q_{a}\right)_{i_{1} i_{2}}=\left[Q_{a}^{g}, W_{0, i_{1} i_{2}}\right]+W_{0,\left(i_{1}\right.}\left[W_{\left.0, i_{2}\right)}, Q_{a}^{g}\right] \\
& \left(\bar{q}_{a}\right)=Q_{a}^{g} \\
& \left(\bar{q}_{a}\right)_{i_{1}}=-\left[Q_{a}^{g}, W_{0, i_{1}}\right] \\
& \left(\bar{q}_{a}\right)_{i_{1} i_{2}}=\partial_{\left(i_{1}\right.}\left[W_{\left.0, i_{2}\right)}, Q_{a}^{g}\right]+\left[Q_{a}^{g}, W_{0, i_{i} i_{2}}\right]+W_{0,\left(i_{1}\right.}\left[W_{\left.0, i_{2}\right)}, Q_{a}^{g}\right] .
\end{aligned}
$$

For the invariant non-local conserved quantities $Q_{a}(\bar{u}, u)=F^{-1}(\bar{u}, u) Q_{a}^{g} F(\bar{u}, u)$ in $(\mathrm{A} .3 \mathrm{~m})$, we find the analogous results,

$$
\begin{gathered}
Q_{a}(\bar{u}, u)=\sum_{p=0}^{\infty} \frac{(u-\bar{u})^{p}}{p !}\left(q_{a}\right)_{p}(\bar{u})=\sum_{p=0}^{\infty} \frac{(\bar{u}-u)^{p}}{p !}\left(\bar{q}_{a}\right)_{p}(u) \\
\left.\left(q_{a}\right)_{p}(\bar{u}) \equiv \partial^{p} Q_{a}(\bar{u}, u)\right|_{u=\bar{u}} \quad,\left.\quad\left(\bar{q}_{a}\right)_{p}(u) \equiv \bar{\partial}^{p} Q_{a}(\bar{u}, u)\right|_{\bar{u}=u} \\
\left(q_{a}\right)_{p}(\bar{u})=\sum_{r=0}^{\infty}(-)^{r} \sum_{p_{1}=1}^{\infty} \cdots \sum_{p_{r}=1}^{\infty} \sum_{s=0}^{\infty} \delta\left(p-s-\sum_{i=1}^{r} p_{i}\right) \frac{p !}{s ! \prod_{i=1}^{r} p_{i} !} \\
\times W_{0 p_{1}} W_{0 p_{2}} \cdots W_{0 p_{r}} Q_{a}^{g} W_{0, s+1} \\
\left(q_{a}\right)_{0}=Q_{a}^{g}
\end{gathered}
$$




$$
\begin{gathered}
\left(q_{a}\right)_{1}=\left[Q_{a}^{g}, W_{01}\right] \\
\left(q_{a}\right)_{2}=\left[Q_{a}^{g}, W_{02}\right]+2 W_{01}\left[W_{01}, Q_{a}^{g}\right] \\
\left(\bar{q}_{a}\right)_{0}=Q_{a}^{g} \\
\left(\bar{q}_{a}\right)_{1}=-\left[Q_{a}^{g}, W_{01}\right] \\
\left(\bar{q}_{a}\right)_{2}=2 \partial\left[W_{01}, Q_{a}^{g}\right]+\left[Q_{a}^{g}, W_{02}\right]+2 W_{01}\left[W_{01}, Q_{a}^{g}\right] .
\end{gathered}
$$

\section{References}

[1] V.G. Kac, Funct. Anal. App. 1 (1967) 328; R.V. Moody, Bull. Am. Math. Soc. 73 (1967) 217.

[2] K. Bardakçi and M.B. Halpern, Phys. Rev. D3 (1971) 2493.

[3] M.B. Halpern and E. Kiritsis, Mod. Phys. Lett. A4 (1989) 1373; Erratum ibid. A4 (1989) 1797.

[4] A.Yu Morozov, A.M. Perelomov, A.A. Rosly, M.A. Shifman and A.V. Turbiner, Int. J. Mod. Phys. A5 (1990) 803.

[5] M.B. Halpern, Phys. Rev. D4 (1971) 2398.

[6] E. Witten, Comm. Math. Phys. 92 (1984) 455; G. Segal, unpublished.

[7] V.G. Knizhnik and A.B. Zamolodchikov, Nucl. Phys. B247 (1984) 83.

[8] P. Goddard, A. Kent and D. Olive, Phys. Lett. B152 (1985) 88.

[9] E. Witten, in Memorial Volume for V. Knizhnik, ed. L. Brink et al., World Scientific, 1990.

[10] M.B. Halpern, E. Kiritsis, N.A. Obers, M. Porrati and J.P. Yamron, Int. J. Mod. Phys. A5 (1990) 2275.

[11] M.B. Halpern, E.B. Kiritsis and N.A. Obers, Proceedings of the RIMS Research Project 1991, "Infinite Analysis", Int. J. Mod. Phys. A7, Suppl. 1 A (1992) 339. 
[12] M.B. Halpern, "Recent Developments in the Virasoro Master Equation", in the proceedings of the Stony Brook conference, Strings and Symmetries 1991, World Scientific, 1992.

[13] M.B. Halpern and N.A. Obers, "Ward Identities for Affine-Virasoro Correlators", Berkeley/Bonn preprint, UCB-PTH-92/24, BONN-HE-92/21, hepth 9207071, 1992. To appear in Int. J. Mod. Phys. A

[14] M.B. Halpern and N.A. Obers, "Solving the Ward Identities of Irrational Conformal Field Theory", Berkeley/Bonn preprint, UCB-PTH93/18, BONN-HE-93/17, hep-th 9305072, 1993. To appear in Int. J. Mod. Phys. A

[15] M.B. Halpern, "Recent Progress in Irrational Conformal Field Theory", Berkeley preprint, UCB-PTH-93/25, hep-th 9309087, 1993. To appear in the proceedings of the Berkeley conference, Strings 1993.

[16] M.B. Halpern, Ann. of Phys. 194 (1989) 247.

[17] M.B. Halpern and N.A. Obers, Nucl. Phys. B345 (1990) 607.

[18] M.B. Halpern and J.P. Yamron, Nucl. Phys. B351 (1991) 333.

[19] M.R. Douglas, "G/H Conformal Field Theory", Caltech preprint, CALT68-1453, 1987, unpublished.

[20] M.Yu. Lashkevich, "Conformal Blocks of Coset Construction: Zero Ghost Number", Landau Inst. preprint, LANDAU-92-TMP-1, 1992. 